\title{
Statistical Mechanics for Truncations of the Burgers-Hopf Equation: A Model for Intrinsic Stochastic Behavior with Scaling
}

\author{
A. Majda and I. Timofeyev
}

\begin{abstract}
In this paper we consider both analytically and numerically several finite-dimensional approximations for the inviscid Burgers-Hopf equation. Fourier Galerkin truncation is introduced and studied as a simple one-dimensional model with intrinsic chaos and a well-defined mathematical structure allowing for an equilibrium statistical mechanics formalism. A simple scaling theory for correlations is developed that is supported strongly by the numerical evidence. Several semi-discrete difference schemes with similar mathematical properties conserving discrete momentum and energy are also considered. The mathematical properties of the difference schemes are analyzed and the behavior of the difference schemes is compared and contrasted with the Fourier Galerkin truncation. Numerical simulations are presented which show similarities and subtle differences between different finite-dimensional approximations both in the deterministic and stochastic regimes with many degrees of freedom.
\end{abstract}

\section{Introduction}

One challenging common feature of several important problems in contemporary science ranging from short term climate prediction for the coupled atmosphere-ocean systems [1], [2], [3] to simulating protein folding through molecular dynamics [4], [5] is the important fact that larger scale features 
have longer correlation times and are more predictable than the general smaller scale and shorter time scale features of these systems with a huge number of degrees of freedom. For these intuitive reasons, for example, many features of the climate are much more predictable than the weather at a fixed location. Such circumstances naturally suggest the development of suitable stochastic modeling procedures for the reduced systems involving only the degrees of freedom with longer correlation times. Systematic mathematical strategies to treat such issues have been developed very recently in different contexts [4], [6], [7]. Here we study simple one-dimensional systems of equations with such features as highly simplified models for such behavior. Straightforward numerical experiments with these deterministic systems presented below establish that they have intrinsic stochastic dynamics with many degrees of freedom and longer correlation times on the larger scales with general features that can be predicted a-priori through simple mathematical arguments and scaling theories. Thus, such models provide a simple unambiguous test problem for stochastic modeling strategies for treating unresolved degrees of freedom.

The models which are studied here are the Fourier Galerkin truncated spectral approximation to the Hopf or inviscid Burgers equation,

$$
u_{t}+\frac{1}{2}\left(u^{2}\right)_{x}=0
$$

as well as suitable spatially discrete finite-difference approximations to (1.1) which conserve both momentum and energy simultaneously. Various approximations to the equation in (1.1) have a long history. With various dissipative terms added to the right-hand side, this equation becomes both a model for shock dynamics [8] and turbulence theory [9] which can be solved exactly [10] with extremely predictable behavior associated with shock formation and propagation. For suitable dispersive terms added to (1.1), the equations become completely integrable once again with highly predictable and recurrent behavior [8], [11]. Goodman and Lax [12] have shown that the simplest naive dispersive difference approximation to (1.1) has completely integrable behavior for suitable initial data. In contrast, the approximations to (1.1) introduced here have intrinsic stochastic dynamics with strong numerical evidence for ergodicity and mixing as well as scaling behavior so that the larger scales are more predictable with longer correlation times provided that these discrete systems have suitably many degrees of freedom. 
The study of various approximations to the Burgers-Hopf equations in (1.1) has a prominent history involving the symbiotic interaction of mathematical theory and scientific computing to gain insight. Prominent examples of such interaction are the pioneering discovery through numerical experiments of clean soliton interaction for the KdV equation by Zabusky and Kruskal [14] and the related studies of the properties of suitable dispersive difference approximation to (1.1) by Goodman and Lax [12] and Levermore and Liu [20]. Discrete dissipative approximations to (1.1) also have a prominent historical role in the design of numerical methods for computing shock dynamics in much more complicated problems such as gas dynamics, combustion, and magneto-hydrodynamics. For a review, see the article by Harten [21].

This article is written in the style of research described in the preceding paragraph where mathematical theory and scientific computing mingle to give insight on a simplified model, used as a prototype for behavior in vastly more complex systems of interest in contemporary science. This is a prominent research "modus operandi" for modern applied mathematics. For more discussion and recent examples of this mode of research, see the article by the first author [22].

Here is a brief preview of the remainder of this paper. In Section 2 we briefly summarize the structural properties of systems of ODE's which allow for an equilibrium statistical mechanics formulation. The mathematical properties of the Fourier Galerkin approximation to the Burgers-Hopf equation which yield an equilibrium statistical theory and statistical predictions for scaling behavior of correlations are presented in Section 3. Several illuminating exact solutions of the Galerkin approximation to (1.1) also are discussed in that section. The material in Section 3 is an expanded version of the discussion in the authors' very recent paper [19]. Section 4 contains numerical evidence for ergodicity and correlation scaling for Galerkin truncations which strongly confirms the predictions of the theory in Section 3. All of the results presented in Section 4 are completely new. In [19], the authors gave numerical evidence validating the statistical predictions with the classical deterministic initial data, $u_{0}=2 \sin (x)$, with fifty discrete Fourier modes. In Section 4 several new results are presented: 1) confirmation of the statistical predictions with random initial data in a more difficult larger variance regime together with universal behavior as the number of degrees of freedom increases systematically; 2) the transition from completely integrable to chaotic to completely ergodic behavior for Galerkin truncations 
with few degrees of freedom; 3) the route in time from very small perturbations of completely integrable exact solutions with short wavelength spatial structure to a temporal regime with ergodicity, mixing and correlation scaling.

How universal are the results obtained in Section 3 and 4 for Galerkin truncations of the Burgers-Hopf equation? One way to address this issue is to study other suitable discrete approximations to (1.1) which fit the statistical framework summarized in Section 2. Natural candidates for such approximations involve appropriate spatially discrete finite-difference schemes for (1.1) which conserve both discrete momentum and energy. This is the topic of Section 5. In the first part of Section 5, the authors introduce a new simple five-point finite-difference scheme which is consistent with (1.1) and also conserves both discrete momentum and energy. This difference scheme is structurally close to the simple scheme utilized by Goodman and Lax [12] but has radically different mathematical properties as we discuss in Section 5. Goodman and Lax showed that their difference scheme is completely integrable for positive initial data. We briefly review the elementary part of their argument in Section 5 and then give explicit solutions which rigorously demonstrate that the difference scheme from [12] has many solutions which blow-up in finite time for initial data changing sign. We end Section 5 by utilizing exact nonlinear solutions of difference schemes which blow-up in finite time as a criterion to motivate the difference scheme for (1.1) of Zabusky and Kruskal [14]. This difference scheme provides a second example which conserves both discrete momentum and energy. In Section 6 , discrete Fourier series are utilized to compare and contrast the statistical and structural properties of the new five-point finite difference scheme and Kruskal-Zabusky finite-difference scheme in physical space from Section 5 and the Fourier space Galerkin truncation from Section 3. We establish that these methods actually have different nonlinear dynamics at low wavenumbers even as $\Delta x \rightarrow 0$ than the Fourier Galerkin truncation! Finally, numerical evidence for ergodicity and mixing for both the five-point and the Kruskal-Zabusky schemes is presented in Section 7 including a comparison with related numerical results for the Fourier Galerkin truncation method from Section 3. 


\section{A Brief Summary of Equilibrium Statistical Mechanics for ODE's}

In this paper, we will consider various systems of ordinary differential equations (ODE's) given by

$$
\frac{d}{d t} \vec{w}(t)=\vec{F}(\vec{w})
$$

with $\vec{w}=\left(w_{1}, \ldots, w_{N}\right)$ and $\vec{F}=\left(F_{1}(\vec{w}), \ldots, F_{N}(\vec{w})\right)$. These ODE's will be suitable discretizations of (1.1) in either Fourier space or physical space. The approximations which we study will always conserve a discrete form of the energy, so that

$$
E(\vec{w})=\frac{1}{2} \sum_{j=1}^{N}\left|w_{j}\right|^{2}
$$

is a conserved quantity for the solution of (2.1). The key property for (2.1) needed to setup an equilibrium statistical mechanics theory is the Liouville property, i.e. the vector field $\vec{F}$ satisfies

$$
\operatorname{div} \vec{F}=\sum_{j=1}^{N} \frac{\partial F_{j}}{\partial w_{j}}=0 .
$$

Associated with the ODE's in (2.1) is the Liouville equation for the transport of probability measures involving statistical ensembles of solutions of (2.1) given by

$$
\begin{aligned}
\frac{\partial f}{\partial t}+\sum_{j=1}^{N} \frac{\partial}{\partial w_{j}}\left[F_{j}(\vec{w}) f\right] & =0 \\
\left.f\right|_{t=0} & =f_{0} .
\end{aligned}
$$

In (2.4), $f_{0}$ is the density on $R^{N}$ of the initial probability measure so that

$$
f_{0} \geq 0 \text { and } \int_{R^{N}} f_{0}=1 .
$$

The Liouville property in (2.3) guarantees that any smooth function of $E$, $G(E)$, is automatically an equilibrium or steady state solution of (2.4).

The natural measures on $R^{N}$ for making measurements for equilibrium statistical mechanics are the canonical Gibbs measures with density given by

$$
G_{\beta}(\vec{w})=C_{\beta} e^{-\beta E(\vec{w})}, \quad \beta>0
$$


where $C_{\beta}$ is a positive normalizing constant picked to guarantee that $G_{\beta}(\vec{w})$ is a probability measure. With the property in (2.3), $G_{\beta}$ is a steady state solution of the Liouville equation in (2.4). The canonical Gibbs measures in (2.6) are the unique probability measures which maximize over all probability densities the information theoretic entropy

$$
S(p)=-\int_{R^{N}} p \ln (p) d w, \quad p \geq 0, \int_{R^{N}} p=1
$$

subject to the mean energy constraint

$$
\bar{E}=\int_{R^{N}} E(\vec{w}) p d w .
$$

Here $\bar{E}$ is a given positive constant and $\beta$ is the Lagrange multiplier associated with this constraint (see [23] for example).

\section{The First Model: Galerkin Truncation of the Burgers-Hopf Equation}

To develop the approximation to (1.1) which defines this model, let $P_{\Lambda} f=$ $f_{\Lambda}$ denote the finite Fourier series truncation of $f$,

$$
P_{\Lambda} f=f_{\Lambda}=\sum_{|k| \leq \Lambda} \hat{f}_{k} e^{i k x} .
$$

Here and elsewhere in the paper it is tacitly assumed that $f$ is $2 \pi$-periodic and real-valued so that the complex Fourier coefficients $\hat{f}_{k}$ satisfy $\hat{f}_{-k}=\hat{f}_{k}^{*}$. The positive integer, $\Lambda$, from (3.1) defines the number of complex-valued degrees of freedom in the approximation. With these preliminaries, the model introduced and studied here is the approximation to (1.1)

$$
\left(u_{\Lambda}\right)_{t}+\frac{1}{2} P_{\Lambda}\left(u_{\Lambda}^{2}\right)_{x}=0 .
$$

This is the Fourier Galerkin truncated approximation to (1.1). With the expansion

$$
u_{\Lambda}(t)=\sum_{|k| \leq \Lambda} \hat{u}_{k}(t) e^{i k x}, \quad \hat{u}_{-k}=\hat{u}_{k}^{*}
$$

the equations in (3.2) can be written equivalently as the following system of nonlinear ordinary differential equations for the amplitudes $u_{k}(t)$ with 
$|k| \leq \Lambda$

$$
\frac{d}{d t} \hat{u}_{k}=-\frac{i k}{2} \sum_{\substack{k+p+q=0 \\|p|,|q| \leq \Lambda}} \hat{u}_{p}^{*} \hat{u}_{q}^{*} .
$$

It is elementary to show that solutions of the equations in either (3.2) or (3.4) have conservation of both momentum and energy, i.e.

$$
M=\frac{1}{2 \pi} \int_{0}^{2 \pi} u_{\Lambda}(t)=\hat{u}_{0}
$$

and

$$
E=\frac{1}{4 \pi} \int u_{\Lambda}^{2} d x=\frac{1}{2}\left|\hat{u}_{0}\right|^{2}+\sum_{k=1}^{\Lambda}\left|\hat{u}_{k}\right|^{2}
$$

are constant in time for solutions of (3.2) or (3.4). The proof for conservation of energy is as follows:

$$
\frac{\partial}{\partial t} \int u_{\Lambda}^{2}=-\frac{1}{2} \int u_{\Lambda} P_{\Lambda}\left(u_{\Lambda}^{2}\right)_{x}=-\frac{1}{2} \int u_{\Lambda}\left(u_{\Lambda}^{2}\right)_{x}=-\frac{1}{3} \int\left(u_{\Lambda}^{3}\right)_{x}=0 .
$$

The momentum constraint in (3.5) is associated with trivial dynamical behavior and without loss of generality, we set $M=0$, so that $\hat{u}_{0}(t) \equiv 0$ in the formula for the energy, $E$, in (3.6). Also, all of the sums in (3.4) involve only $k$ with $1 \leq k \leq \Lambda$. In addition to the conserved quantities in (3.5) and (3.6) the discrete analog

$$
\int u_{\Lambda}^{3} d x
$$

is also conserved. Gregor Kovacic and the authors have shown that the dynamics in (3.2) or (3.4) is a noncanonical Hamiltonian system with the same symplectic structure as arises for the Korteweg deVries equation. The implications of this fact for the dynamics and statistical behavior will be developed elsewhere by Kovacic and the authors in the near future.

It is well known that nontrivial smooth solutions of (1.1) develop discontinuities in finite time and thus exhibit a transfer of energy from large scales to small scales. For functions with $\hat{u}_{k}(t)$ identically zero instantaneously for $k>\frac{\Lambda}{2}$ the approximation in (3.2) or (3.4) represents this energy transfer exactly; however, once this transfer develops in a general solution of (3.2) or (3.4), the conservation of energy constraint rapidly redistributes the energy in the smaller scales to the the larger scale modes. This effect is 
responsible for the intuitive fact that the small scale modes of the system should decorrelate more rapidly than the large scale modes.

\subsection{Equilibrium Statistical Mechanics for the Model}

It is natural to utilize the conserved quantity given by the energy, $E$, in (3.6) to define an invariant Gibbs measure for equilibrium statistical mechanics as discussed in Section 2. In order to be able to do this, the ordinary differential equations in (3.4) need to satisfy the Liouville property in (2.3). As mentioned in the preceding paragraph, the equations in (3.4) have individual solutions which exhibit compressible transfer of energy to the smaller scales. Nevertheless, the equations in (3.4) satisfy the Liouville property for statistical solutions; to see this, since $\hat{u}_{0}=0$ and $\hat{u}_{-k}=\hat{u}_{k}^{*}$, the modes $\hat{u}_{k}=a_{k}+i b_{k}$ with $1 \leq k \leq \Lambda$ are the defining modes for the equations in (3.4). The equation for $\hat{u}_{k}(t)$ in (3.4) can be written in the following form in terms of $a_{k}$ and $b_{k}$

$$
\frac{d}{d t}\left(\begin{array}{c}
a_{k} \\
b_{k}
\end{array}\right)=\left(\begin{array}{c}
V_{k}^{1} \\
V_{k}^{2}
\end{array}\right)=\left(\begin{array}{c}
2 k\left(b_{k} a_{-2 k}+a_{k} b_{-2 k}\right) \\
-2 k\left(a_{k} a_{-2 k}+b_{k} b_{-2 k}\right)
\end{array}\right)
$$

plus the terms without $a_{k}, b_{k}$.

Since $\partial V_{k}^{1} / \partial a_{k}+\partial V_{k}^{2} / \partial b_{k}=0$ as follows from (3.8) the Liouville property is satisfied. With this property, the canonical Gibbs measures

$$
G_{\beta}=C_{\beta} \operatorname{EXP}\left(-\beta \sum_{k=1}^{\Lambda}\left|\hat{u}_{k}\right|^{2}\right), \quad \beta>0
$$

are invariant probability measures for the statistical dynamics of eq. (3.2) or (3.4). Given a value for the mean energy, $\bar{E}$, from (3.6), $\beta$ is given by

$$
\beta=\frac{\Lambda}{\bar{E}}, \text { with } \operatorname{Var}\left\{a_{k}\right\}=\operatorname{Var}\left\{b_{k}\right\}=\frac{1}{2 \beta}
$$

where $V a r$ denotes the variance. The canonical Gibbs ensemble predicts a spectrum with equipartition of energy in all modes according to (3.10). We show below that these statistical predictions are satisfied with surprising accuracy for $\Lambda$ of moderate size. 


\section{A Scaling Theory for Temporal Correlations}

It is a simple matter to present a scaling theory which predicts that the temporal correlation times of the large scale modes are longer than those for the small scale modes. Recall from (3.10) that the statistical predictions for the energy per mode is $\bar{E} / \Lambda=\beta^{-1}$; since $\bar{E} / \Lambda$ has units length ${ }^{2} / \mathrm{time}^{2}$, and the wavenumber $k$ has units length ${ }^{-1}$ the predicted eddy turnover time for the $k$-th mode is given by

$$
\left(\frac{\Lambda}{\bar{E}}\right)^{1 / 2} \frac{1}{k}=\frac{\sqrt{\beta}}{k}, \quad 1 \leq k \leq \Lambda .
$$

If the physical assumption is made that the $k$-th mode decorrelates on a timescale proportional to the eddy turnover time with a universal constant of proportionality, $C_{0}$, a simple plausible scaling theory for the dynamics of the equation in (3.2) or (3.4) emerges and predicts that the correlation time for the $k$-th mode, $T_{k}$, is given by

$$
T_{k}=\frac{C_{0} \sqrt{\beta}}{k}, \quad 1 \leq k \leq \Lambda .
$$

Thus, the scaling theory implied by (3.11) shows that the larger scale modes in the system should have longer correlation times than the smaller scale modes. This basic qualitative fact is always confirmed in the numerical simulations for the Fourier Galerkin truncation. The exact quantitative agreement of computed correlation times with the predictions of the scaling theory is also reported below in Section 4 and for another case in [19].

\subsection{Invariant Low Dimensional Subspaces}

Virtually all inhomogeneous systems with many degrees of freedom and intrinsic stochastic behavior also possess lower dimensional invariant sets with non-generic and atypical dynamic behavior. Here we show how to construct large families of lower dimensional invariant subspaces for the dynamics in (3.2) or (3.4).

To build these invariant subspaces, pick any positive integer $k_{*}$ satisfying $2 \leq k_{*} \leq \Lambda$ and consider the unique integer $N$ with

$$
N k_{*} \leq \Lambda<(N+1) k_{*}
$$


Associated with $k_{*}$, we build exact solutions of (3.4) with non-zero Fourier coefficients only at $\hat{u}_{ \pm j k_{*}}$ for $1 \leq j \leq N$, i.e. define $\tilde{u}_{j}$ by

$$
\begin{gathered}
\hat{u}_{ \pm j k_{*}}=\tilde{u}_{ \pm j}, \quad 1 \leq j \leq N \\
\hat{u}_{k}=0, \quad k \neq \pm j k_{*} .
\end{gathered}
$$

Functions with Fourier coefficients satisfying the symmetries in (3.13) are not only $2 \pi$-periodic but are also spatially periodic with the smaller period, $2 \pi / k_{*}$. For elegance in notation, given $k_{*}$, we rescale time to the faster time scale $\tau=k_{*} t$, then a short calculation confirms that the non-zero Fourier coefficients in (3.13) satisfy the same dynamical equations in (3.4) with $2 N$ degrees of freedom which are strictly less than $2 \Lambda$ degrees of freedom in the general solution of the original system, i.e.

$$
\frac{d}{d \tau} \tilde{u}_{k}=-\frac{i k}{2} \sum_{\substack{k+p+q=0 \\|p|,|q| \leq \Lambda}} \tilde{u}_{p}^{*} \tilde{u}_{q}^{*}, \quad \text { for }|k| \leq N
$$

with $\hat{u}_{k}(t)=0$ for $k \neq \pm j k_{*}$.

For $k_{*}$ with $\frac{\Lambda}{2} \leq k_{*} \leq \Lambda, N=1$ and the equations in (3.14) trivially yield the time independent steady state

$$
\begin{gathered}
u_{k_{*}}=u_{k}^{0}, \quad \text { any complex constant } \\
u_{k}=0 \quad \text { for } k \neq k_{*}, 1 \leq k \leq \Lambda .
\end{gathered}
$$

For $k_{*}$ with $\frac{\Lambda}{3} \leq k_{*}<\frac{\Lambda}{2}, N=2$ and the non-zero Fourier components $\hat{u}_{k_{*}}, \hat{u}_{-2 k_{*}}$ are defined through (3.14) with $N=2$, i.e.

$$
\begin{gathered}
\frac{d}{d \tau} \tilde{u}_{1}=-i \tilde{u}_{1}^{*} \tilde{u}_{-2}^{*} \\
\frac{d}{d \tau} \tilde{u}_{-2}=i\left(\tilde{u}_{1}^{*}\right)^{2} .
\end{gathered}
$$

First, note that equations in (3.16) have the steady state solution defined by the higher wavenumber $\tilde{u}_{-2}=\tilde{u}_{-2}^{0}, \tilde{u}_{1}=0$ which corresponds to the steady state already mentioned in (3.15). Linearized perturbations $\tilde{u}_{1}^{\prime}$ about this steady state satisfy the equation

$$
\frac{d}{d \tau} \tilde{u}_{1}^{\prime}=-i\left(\tilde{u}_{1}^{\prime}\right)^{*} \tilde{u}_{-2}^{0}
$$

with exponentially growing solution so that these higher wavenumber steady states are dynamically unstable to lower wavenumber perturbations. This 
is the simplest example exhibiting the tendency of the equations in (3.2) or (3.4) to transfer energy to larger scales.

In fact, the equations with $N=2$ are completely integrable since conservation of energy implies that $\left|\tilde{u}_{1}\right|^{2}=E-\left|\tilde{u}_{2}\right|^{2}$ and differentiating the second equation in (3.16) yields

$$
\frac{d^{2}}{d \tau^{2}} \tilde{u}_{-2}=-2\left|\tilde{u}_{1}\right|^{2} \tilde{u}_{-2}=-2\left(E-\left|\tilde{u}_{2}\right|^{2}\right) \tilde{u}_{-2} .
$$

Following the discussion in [13] we show that the equation in (3.18) is integrable so the dynamics for $N=2$ has regular non-chaotic behavior. We make the change of variables

$$
\hat{u}(t)_{-2}=r(t) e^{i \phi(t)}
$$

and substitute (3.19) into the equation in (3.18) and separately equate real and imaginary part to zero. First, from the imaginary part we obtain

$$
2 \dot{r} \dot{\phi}+r \ddot{\phi}=0
$$

where $\left\{\dot{\}}\right.$ denotes the time-derivative, i.e. $\left\{\dot{\}}=\frac{d}{d t}\right.$. The equation in (3.20) implies that

$$
\frac{d}{d t}\left(r^{2} \dot{\phi}\right)=0
$$

i.e. $r^{2} \dot{\phi}$ is conserved in time

$$
r^{2} \dot{\phi}=C .
$$

Second, from the real part we obtain

$$
\ddot{r}-r \dot{\phi}^{2}=-2\left(E-r^{2}\right) r
$$

and expressing the time-derivative $\dot{\phi}$ from (3.22) we obtain the second order differential equation for $r$ alone

$$
\ddot{r}-\frac{C^{2}}{r^{3}}=-2\left(E-r^{2}\right) r .
$$

Multiplying the equation in (3.24) by $\dot{r}$ we immediately recognize that

$$
\mathcal{E}=\frac{1}{2}\left(\frac{d r}{d t}\right)^{2}+V(r)
$$

is constant, where

$$
V(r)=E r^{2}+\frac{1}{2} \frac{C^{2}}{r^{2}}-\frac{1}{2} r^{4} .
$$

The conserved quantity in (3.25) is analogous to the energy equation of a particle, whose position is $r(t)$, subject to the force potential $V(r)$ and the total energy $\mathcal{E}$, which is fixed by values of $\dot{r}$ and $r$ at the initial time. The 
transition from complete integrability to ergodic behavior with mixing as $N$ is increased through $N=3,4,5$ is one of the topics of the next section.

\section{Numerical Evidence for Ergodicity and Correlation Scaling}

In all the numerical simulations presented in this section, a pseudo-spectral method of spatial integration combined with fourth order Runge-Kutta time stepping is utilized for (3.4). One can anticipate from (3.9), which predicts energy equipartition, that in dynamic simulations both the high and low spatial wavenumbers are equally important so increased spatial resolution in the pseudo-spectral algorithm is needed. This is achieved by increasing the length of the array containing the discrete Fourier coefficients by adding zeros for wavenumbers $k$ with $\Lambda<k \leq \Lambda_{*}$ and then performing the discrete Fourier transform to $x$-space on this bigger array for pseudospectral computations. The choice $\Lambda_{*} \geq 4 \Lambda$ works well in practice and is utilized below. The typical time step for the Runge-Kutta time integrator is $\Delta t=2 \times 10^{-4}$, with the necessity to use smaller time steps for larger values of the total energy $\bar{E}$ (smaller $\beta$ and/or larger $\Lambda$ ). In all simulations presented below the energy computed from (3.6) is conserved within $10^{-4}$ percent, a relative error of $10^{-6}$.

All statistical quantities are computed as time-averages. In particular, the energy in the $k$-th mode is computed by

$$
\left\langle\left|\hat{u}_{k}\right|^{2}\right\rangle=\frac{1}{T} \int_{T_{0}}^{T+T_{0}}\left|\hat{u}_{k}(t)\right|^{2} d t .
$$

This is a severe test because only the microcanonical statistics for an individual solution of (3.4) are utilized rather than a Monte-Carlo average over many random initial data as given by the canonical Gibbs ensemble in (3.9). The time correlations $\left\langle\operatorname{Re} \hat{u}_{k}(t+\tau) \operatorname{Re} \hat{u}_{k}(\tau)\right\rangle$ are computed by the same numerical averaging procedure from (4.1).

The initial data is selected at random with Fourier coefficients $u_{k}$, $1 \leq k<\Lambda-15$ sampled from a Gaussian distribution with mean zero and variance $0.8 \times \beta^{-1}$. The tail Fourier coefficients with $\Lambda-15 \leq k \leq \Lambda$ are 
initialized with random phases and equal amplitudes

$$
\left|u_{k}\right|^{2}=\frac{1}{15}\left(\bar{E}-\sum_{j=1}^{\Lambda-16}\left|u_{j}\right|^{2}\right), \quad \Lambda-15 \leq k \leq \Lambda
$$

to satisfy the energy constraint given by $\bar{E}$.

A wide range of simulations of (3.4) have been performed in several parameter regimes. A detailed numerical study of the equations in (3.4) with $\beta=50, \Lambda=50$ was already reported in [19]. In particular, simulations with the deterministic initial data $u_{0}=2 \sin (x)$ were discussed and compared with simulations with random initial data. Numerical results presented in [19] provide convincing evidence for the validity of the theoretical predictions of the equilibrium statistical mechanics and the scaling theory for correlations and also for the ergodicity of the dynamics of the equations in (3.4). Here we discuss a more difficult regime both computationally and numerically with the larger variance $\beta=10$ and $\Lambda$ varying over 50, 100, 200 . All three of these truncations exhibit qualitatively similar behavior and we focus our attention on the numerical simulations with $\beta=10, \Lambda=100$. Some results of the simulations with $\Lambda=50$ and $\Lambda=200$ are presented for comparison and to emphasize the dependence of the statistical quantities on the number of Fourier modes in the truncation, $\Lambda$.

The numerical results discussed in this section are qualitatively similar to the simulations with $\Lambda=50, \beta=50$ described in [19]. In particular, the equipartition of the spectrum and the agreement with the predictions of the scaling theory for temporal correlations for modes with $k \leq 15$ were observed in all simulations with surprising accuracy. The results of the numerical simulations presented in this section support the numerical evidence about the qualitatively universal behavior of the equations in (3.4). In the simulation presented here, the initial averaging value, $T_{0}=100$, and averaging window, $T=5000$, were utilized.

The energy spectrum for the real and imaginary parts of the Fourier modes is presented in the top and the bottom parts of Figure 4.1, respectively. The straight lines in Figure 4.1 correspond to the theoretically predicted value $\operatorname{Var}\left\{\operatorname{Re} \hat{u}_{k}\right\}=\operatorname{Var}\left\{\operatorname{Im} \hat{u}_{k}\right\}=0.05$ with $\beta=10$. Clearly, there is statistical equipartition of energy for times $t$ with $t \geq 100$ in the solution of (3.4); the relative errors mostly occur at large scales and do not exceed $3.5 \%$.

The time correlations of Re $\hat{u}_{k}$ for $k=1,2,3,10,15,20$ are presented in Figure 4.2 illustrating the wide range of time scales present in the system. 

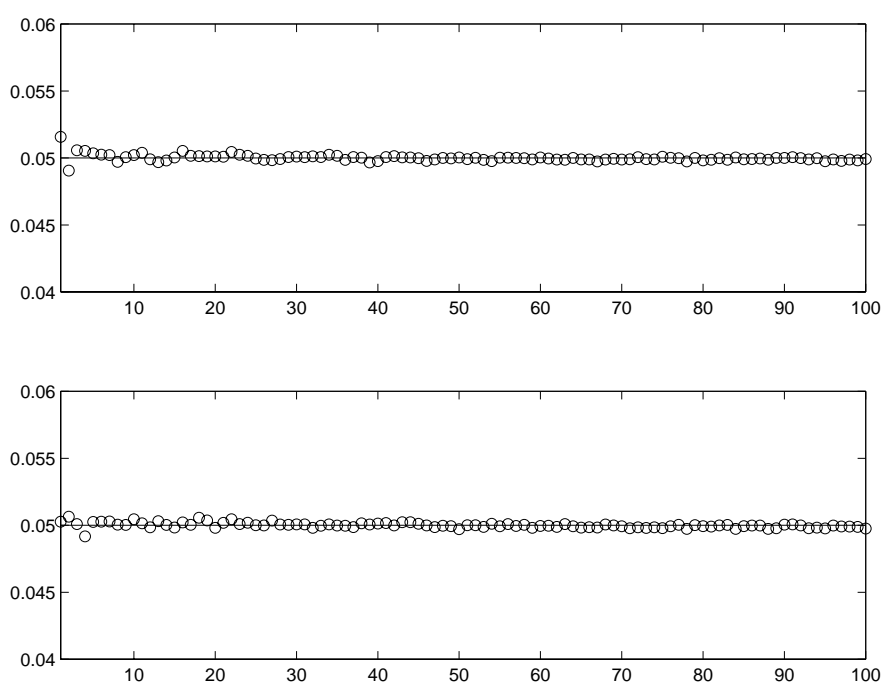

Figure 4.1: Galerkin truncation with $\beta=10, \Lambda=100$. Energy Spectrum; Circles - Numerics, Solid Line - Analytical Predictions.

The elementary scaling theory for correlation times developed in (3.11) is compared with the numerically computed correlation times in Figure 4.3. There the scaling formula in (3.11) is multiplied by a normalization constant, $C_{0}$, to exactly match the correlation time for the mode with $k=1$. As shown in Figure 4.3, the simple theory proposed in (3.11) is an excellent fit for the large scale wavenumbers, $k$ with $k \leq 15$, which exhibit the largest range of scaling behavior for the temporal correlations.

The equipartition of the spectrum and correlation scaling behavior predicted by the theory in (3.9), (3.10), and (3.11) is very robust. The only caveat in this discussion is the normalization constant for the correlation scaling theory in (3.11) which depends upon the correlation time of the largest scale mode with $k=1$.

Correlation functions for mode $k=1$ in the simulations with $\beta=10$ and $\Lambda=50,100,200$ are depicted in Figure 4.4, which illustrates the dependence of the correlation time of the Fourier mode with $k=1$ (and thus, the normalization constant, $C_{0}$, in (3.11)) on $\Lambda$. Table 4.1 summarizes the dependence of the normalization constant on $\beta$ and $\Lambda$ in nine different parameter regimes. 


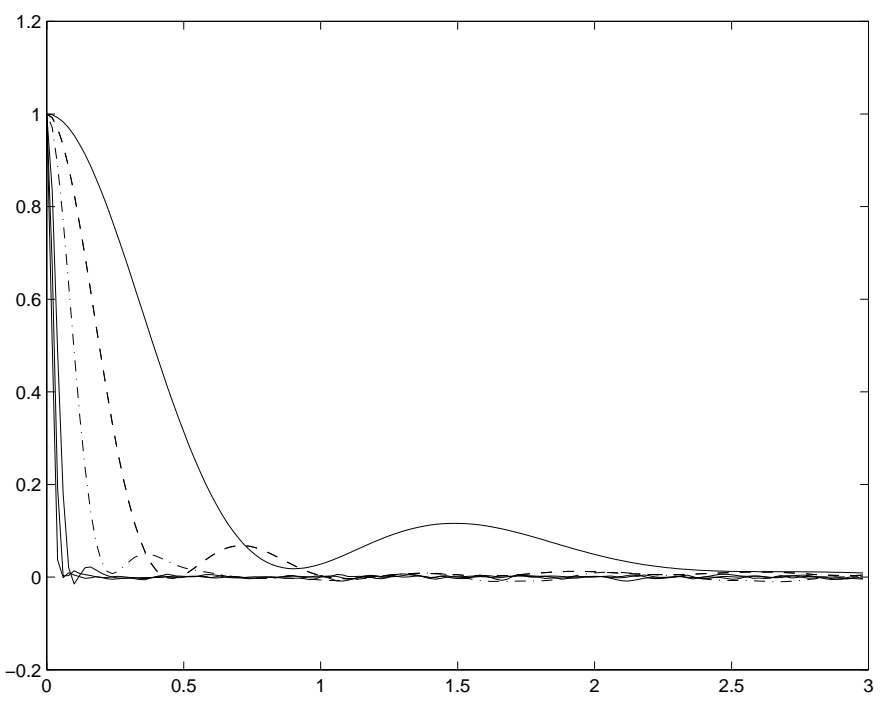

Figure 4.2: Galerkin truncation with $\beta=10, \Lambda=100$. Correlation Functions for modes $k=1,2,3,10,15,20$.

\begin{tabular}{|c|c|c|c|}
\hline$\Lambda \backslash \beta$ & 10 & 50 & 75 \\
\hline 50 & 0.177 & 0.174 & 0.1729 \\
100 & 0.156 & 0.166 & 0.1528 \\
200 & 0.134 & 0.126 & 0.1177 \\
\hline
\end{tabular}

Table 4.1: Normalization Constant, $C_{0}$, in Simulations with $\beta=10,50,75$ and $\Lambda=50,100,200$

The normalization constant, $C_{0}$, is nearly universal for the simulations with varying $\beta$ for the same value of $\Lambda$, but depends weakly on $\Lambda$ for fixed $\beta$; however, correlation times for low and high wavenumbers scale uniformly with $\Lambda$ and the ratio of the largest to the smallest correlation time in the system is independent of $\Lambda$ for all the parameter regimes tested. Correlation times, $T_{k}$, for selected wavenumbers in the three regimes $\beta=10$ and $\Lambda=50,100,200$ are presented in Table 4.2. Correlation times for all modes decrease with $\Lambda$ and the predictions of the scaling theory are confirmed by the numerical simulations in all three parameter regimes. 


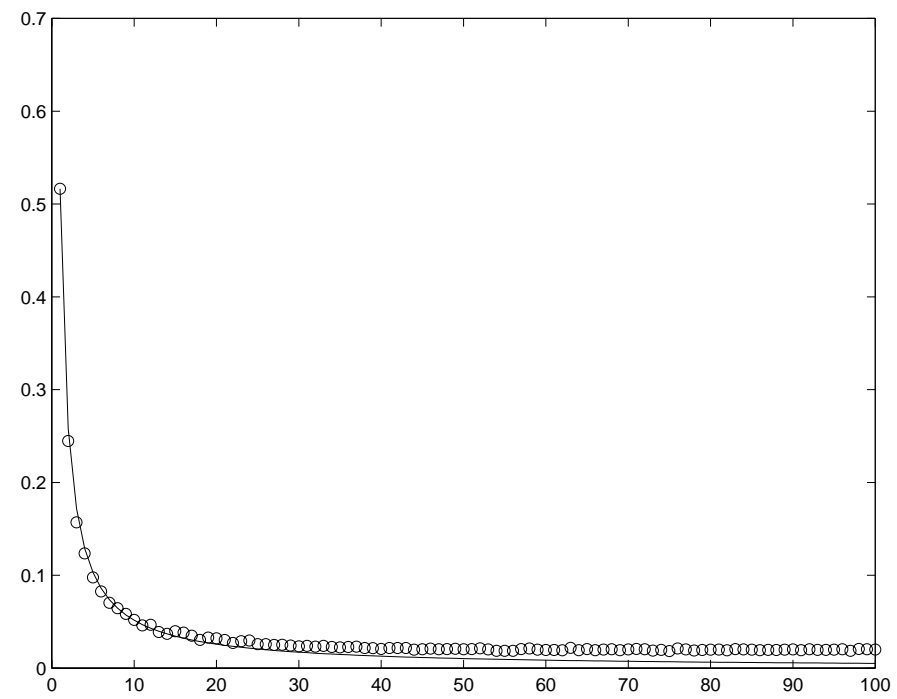

Figure 4.3: Galerkin truncation with $\beta=10, \Lambda=100$. Correlation Times vs wavenumber $k$. Circles - DNS, Solid Line - Predictions of the Scaling Theory.

\begin{tabular}{|c|c|c|c|}
\hline mode $\backslash \Lambda$ & 50 & 100 & 200 \\
\hline 1 & 0.5610 & 0.5164 & 0.4240 \\
2 & 0.2983 & 0.2447 & 0.1934 \\
3 & 0.2055 & 0.1571 & 0.1179 \\
25 & 0.0338 & 0.0258 & 0.0212 \\
50 & 0.0252 & 0.0204 & 0.0203 \\
75 & N/A & 0.0183 & 0.0202 \\
100 & N/A & 0.0200 & 0.0202 \\
150 & N/A & N/A & 0.0200 \\
200 & N/A & N/A & 0.0204 \\
\hline
\end{tabular}

Table 4.2: Correlation Times, $T_{k}$, for selected modes for simulations with $\beta=$ $10, \Lambda=50,100,200$

The statistical predictions of (3.9) go beyond the energy spectrum and also predict Gaussian behavior for the higher moments. Strong numerical evidence supporting the Gaussian nature of the dynamics in (3.4) was presented in [19] for the regimes with $\beta=50, \Lambda=50$. Here we reproduce 


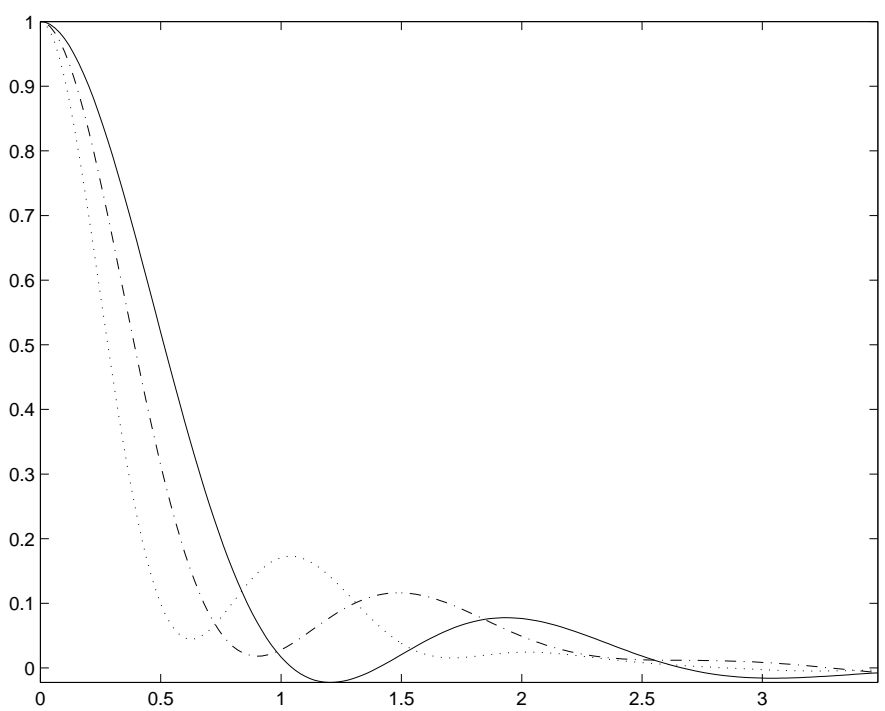

Figure 4.4: Galerkin truncation. Correlation Functions of Re $\hat{u}_{1}$ for runs with $\beta=10$ and $\Lambda=50$ (Solid), 100 (Dashed), 200(Dotted).

results reported earlier in [19] with the larger variance, $\beta=10$, and, also, perform a harder test of Gaussianity of the dynamics of the equations in (3.4), namely the comparison of the eighth moment. Unlike for the regime $\beta=50, \Lambda=50$, dynamic range effects are not present in the regime $\beta=10$, $\Lambda=100$ and we can compute eighth moment with sufficient accuracy. For a Gaussian distribution in (3.9), the following relationships between second and higher moments hold

$$
\begin{gathered}
\left\langle\left|\operatorname{Re} \hat{u}_{k}\right|^{4}\right\rangle=3\left\langle\left|\operatorname{Re} \hat{u}_{k}\right|^{2}\right\rangle^{2}=\frac{3}{4} \beta^{-2} \\
\left\langle\left|\operatorname{Re} \hat{u}_{k}\right|^{6}\right\rangle=15\left\langle\left|\operatorname{Re} \hat{u}_{k}\right|^{2}\right\rangle^{3}=\frac{15}{8} \beta^{-3} \\
\left\langle\left|\operatorname{Re} \hat{u}_{k}\right|^{8}\right\rangle=105\left\langle\left|\operatorname{Re} \hat{u}_{k}\right|^{2}\right\rangle^{4}=\frac{105}{16} \beta^{-4} .
\end{gathered}
$$

To check the Gaussianity of the dynamics we compute the relative error between the analytical predictions in (4.2) and numerical estimates given 
by

Rel. Err. $\{4$ th Moment $\}=\left|\left\langle\left|\operatorname{Re} \hat{\mathrm{u}}_{\mathrm{k}}\right|^{4}\right\rangle-\frac{3}{4} \beta^{-2}\right| \times \frac{4}{3} \beta^{2}$

Rel. Err. $\{6$ th Moment $\}=\left|\left\langle\left|\operatorname{Re} \hat{u}_{k}\right|^{6}\right\rangle-\frac{15}{8} \beta^{-3}\right| \times \frac{8}{15} \beta^{3}$

Rel. Err. $\{$ 8th Moment $\}=\left|\left\langle\left|\operatorname{Re} \hat{u}_{\mathrm{k}}\right|^{8}\right\rangle-\frac{105}{16} \beta^{-4}\right| \times \frac{16}{105} \beta^{4}$.

In Figure 4.5, the relative error in (4.3) for the fourth, sixth, and eighth moments is computed as a function of wavenumber from the simulations of (3.4) with $\beta=10$ and $\Lambda=100$. Relative errors in the fourth moment prediction (the top part of Figure 4.5) are less than $2 \%$ for almost all wavenumbers and never exceed $3.5 \%$ with the largest errors for the low wavenumbers. For the sixth moments (the middle part of Figure 4.5) the relative errors are less than $5 \%$ for most of the wavenumbers and do not exceed $9 \%$ overall. For the eighth moment (the bottom part of Figure 4.5) the relative errors are about $7-8 \%$ for most of the Fourier modes. Thus, the higher order statistics agrees with the predictions of the invariant measure in (3.9) and the Gaussianity of the dynamics of the equations in (3.4) is confirmed with surprising accuracy.
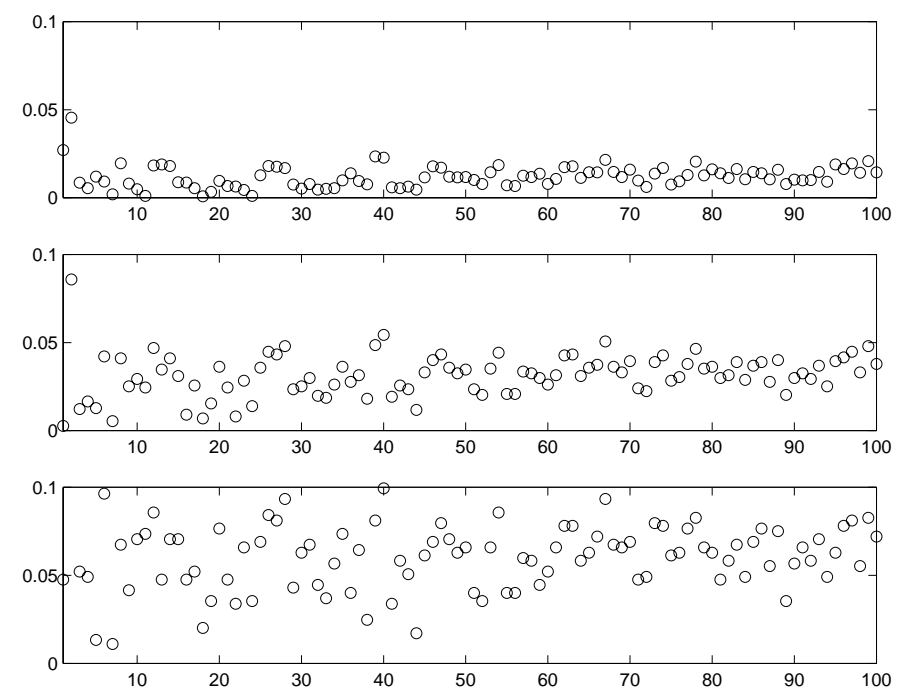

Figure 4.5: Galerkin truncation with $\beta=10, \Lambda=100$. Relative Errors for Numerical and Analytical Estimates for 4th, 6th, and 8th Moments; Vertical axis $=10 \%$ Relative Error. 


\subsection{The Transition from Integrability to Ergodicity with Few Degrees of Freedom}

In this Section we present numerical simulations for the low-dimensional cases with few degrees of freedom so that $N=3,4$, and 5 . Here we use $\Lambda$ and $N$ interchangeably. The initial averaging value, $T_{0}=1000$, and averaging window $T=40000$ were utilized in these simulations. The total energy, $\bar{E}$, is conserved up to $10^{-11}$, relative error $10^{-9}$, in all simulations described in this section. The initial conditions were selected at random in all simulation as follows - the first two Fourier modes $\hat{u}_{1}$ and $\hat{u}_{2}$ were sampled from a Gaussian distribution with mean zero and variance $0.85 \times \beta^{-1}$. Then, the rest of the Fourier coefficients $\hat{u}_{j}, j=3 \ldots \Lambda$ were initialized with random phases and identical amplitudes $\left|\hat{u}_{j}\right|^{2}=\left(\Lambda \beta^{-1}-\left|\hat{u}_{1}\right|^{2}-\left|\hat{u}_{2}\right|^{2}\right)(\Lambda-2)^{-1}$ to satisfy the energy constraint $E=\Lambda / \beta$.

For $N=3$, the dynamics exhibits a mixture of integrable and chaotic behavior. Two simulations with different initial conditions are compared in Figures 4.6 and 4.7. Both initial conditions were selected at random, but the dynamics is drastically different in these two cases. The time series of Re $\hat{u}_{1}$ for two runs are presented in the top parts of Figures 4.6 and 4.7. The top part of Figure 4.6 shows regular quasi-oscillatory behavior, while the top part of Figure 4.7 shows dynamics which resembles chaotic behavior. Correlation functions for Re $\hat{u}_{1}$ for these two runs are presented in the middle parts of Figures 4.6 and 4.7. As expected, the correlation function computed in the quasi-periodic regime (the middle part of Figure 4.6) does not decay at all, while the correlation function in the chaotic regime (the middle part of Figure 4.7) decays to some moderately low values. Nevertheless, the correlation function for the mode with $k=1$ in the chaotic regime does not decay to zero and retains some structure for extremely long times. The set of initial conditions for which the three-dimensional subsystem exhibits chaotic behavior is rather small; most of the initial conditions lead to a regular behavior resembling quasi-periodic motion. The energy spectrum (the bottom parts of Figures 4.6 and 4.7) is not equipartitioned in either simulation and relative errors between numerical and analytical estimates are up to $20 \%$, which is another indication that the dynamics on the three-dimensional subspace is not completely ergodic.

For $N=4$ the situation is the reverse. Most of the initial conditions lead to irregular behavior. Nevertheless, there exist parts of the phase space in which the correlation function for the mode with $k=1$ has a very long tail, which indicates a significant influence of the coherent structures in 

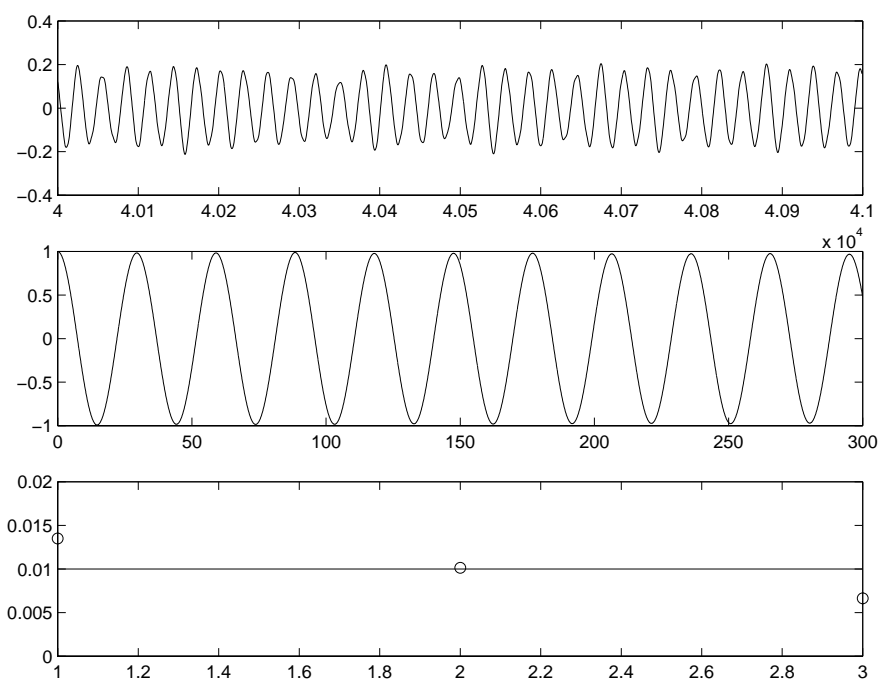

Figure 4.6: Simulations with $N=3$ with regular behavior; Top - Time Series of Re $\hat{u}_{1}$, Middle - Correlation function of Re $\hat{u}_{1}$, Bottom - Averaged Spectrum.

the dynamical system. The time evolution of the Fourier mode Re $\hat{u}_{1}$ for $N=4$, plotted in the top part of Figure 4.8, resembles the oscillatory behavior of the Fourier mode Re $\hat{u}_{1}$ for $N=3$ plotted in the top part of Figure 4.6. The solution for $N=4$ is more chaotic than for $N=3$, but regular behavior is clearly evident in the time series of $\operatorname{Re} \hat{u}_{1}$, which is reflected in the extremely long tail of the correlation function for the mode with $k=1$ shown in the middle part of Figure 4.8.

Numerical and analytical estimates for the energy spectrum are presented in the bottom part of Figure 4.8. Despite the long tail of the correlation function for the mode with $k=1$, we observe a reasonably good agreement with the predictions of the equilibrium statistical mechanics in (3.10). Nevertheless, the energy spectrum is not exactly equipartitioned; the relative errors for the mode with $k=1$ are about $12 \%$ in this simulation which together with slowly decaying correlation functions indicates insufficient dynamic mixing on the four-dimensional space.

Next, we consider $N=5$. The time evolution and correlation function for the mode Re $\hat{u}_{1}$ and the energy spectrum depicted in Figure 4.9 present convincing evidence that the dynamics of the five-dimensional subsystem is completely chaotic. The correlation function for mode with $k=1$ decays 

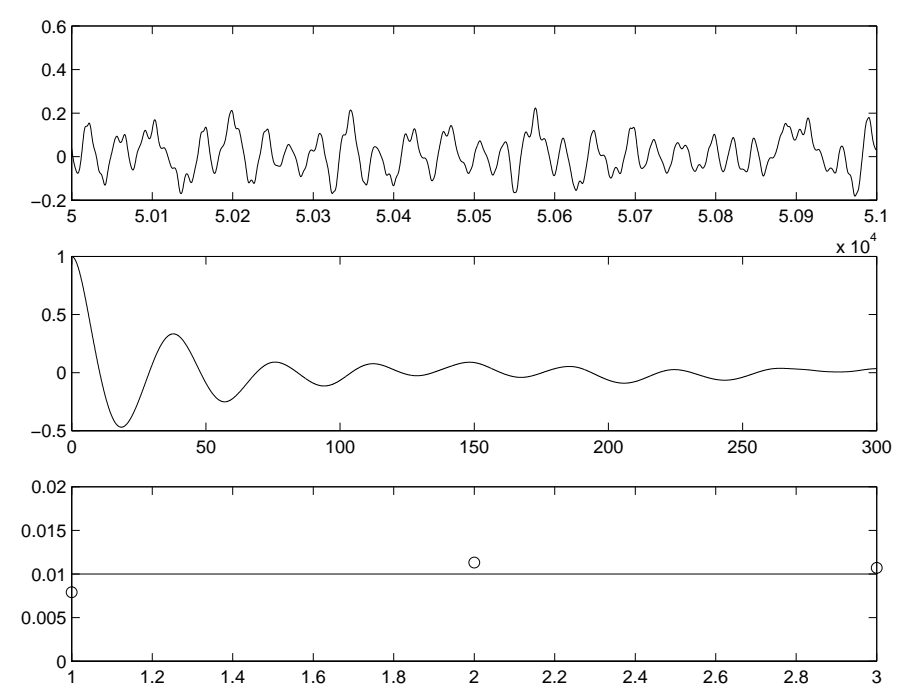

Figure 4.7: Simulations with $N=3$ with irregular, chaotic behavior; Top - Time Series of Re $\hat{u}_{1}$, Middle - Correlation function of Re $\hat{u}_{1}$, Bottom - Averaged Spectrum.

much faster than for $N=4$. The energy spectrum for the simulation with $N=5$ is presented in the bottom part of Figure 4.9. The relative errors in this case are no more than $3.5 \%$ which is consistent with the simulations with larger $\Lambda$ described earlier in Section 4 .

In all simulation tested we found that a chaotic, mixing regime is generic behavior for the equations in (3.4) with $\Lambda \geq 5$. We also found that for any number of degrees of freedom there are sets of initial conditions of measure zero (phase-locked initial condition, completely identical Fourier coefficients, for instance) which lead to a non-generic, more regular behavior with slowly decaying temporal correlations of the Fourier coefficients with low wavenumbers. Thus, not all of the presumed set of measure zero in the complement of the ergodic region consists of the invariant subspaces from 3.2 .

\subsection{Simulations with Initial Conditions as Perturbation of the Integrable Two-Dimensional Invariant Subspace}

In this section the mixing properties of the equations in (3.4) are investigated further by performing numerical simulations with initial data close to the two-dimensional invariant subspace described in Section 3.2. As we have 

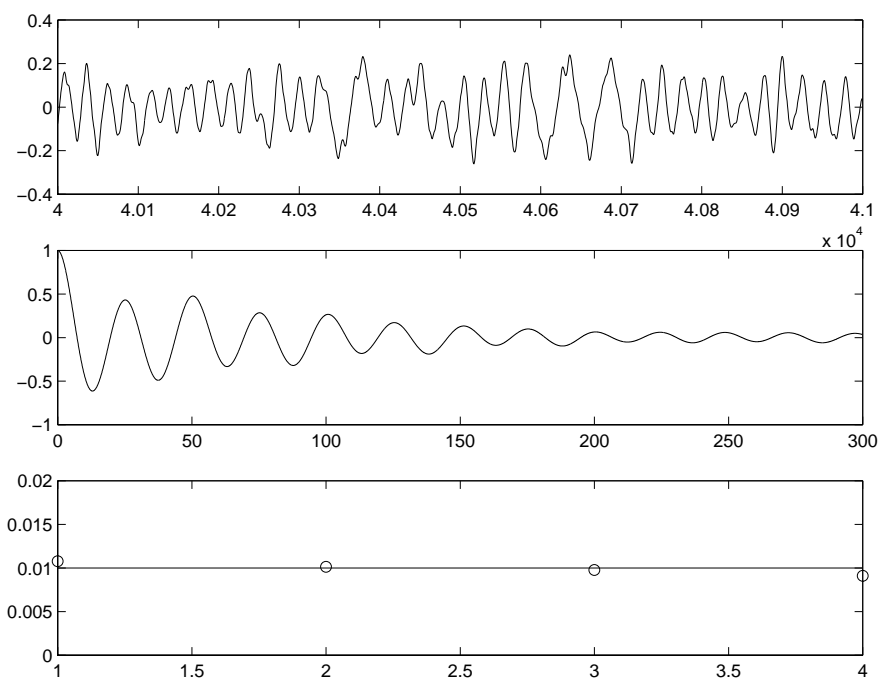

Figure 4.8: Simulations with $N=4$; Top - Time Series of Re $\hat{u}_{1}$, Middle Correlation function of Re $\hat{u}_{1}$, Bottom - Averaged Spectrum.

shown earlier, the two-dimensional invariant subspace is completely integrable, but linearly unstable. Here numerical evidence is presented which shows very strong mixing properties for the equations in (3.4). To contrast with simulations reported in [19] where all the initial energy is contained in the mode with $k=1$ initially (simulations with the initial condition $u_{0}=2 \sin (x)$ ) we perform numerical simulations with $\beta=50$ and $\Lambda=50$ and initial conditions where the short wavelength modes $\hat{u}_{20}$ and $\hat{u}_{40}$ contain $99.9999 \%$ of the total energy at time $t=0$. Amplitudes of the modes $\hat{u}_{20}$ and $\hat{u}_{40}$ are selected to be equal,

$$
\left|\hat{u}_{20}\right|^{2}=\left|\hat{u}_{40}\right|^{2}=0.999999 \times \frac{\bar{E}}{2}=0.999999 \times \frac{1}{2} \beta^{-1} \Lambda, \quad j=20,40,
$$

but the phases are selected at random. The energy of perturbations is contained in the first 19 Fourier modes to allow bigger perturbation per Fourier mode and to avoid the instantaneous inverse cascade at time $t=0$. Fourier coefficients with $1 \leq k<20$ are initialized with equal amplitudes

$$
\left|\hat{u}_{j}\right|^{2}=10^{-6} \times \frac{\bar{E}}{19}=10^{-6} \times \frac{1}{19} \beta^{-1} \Lambda, \quad j<20
$$



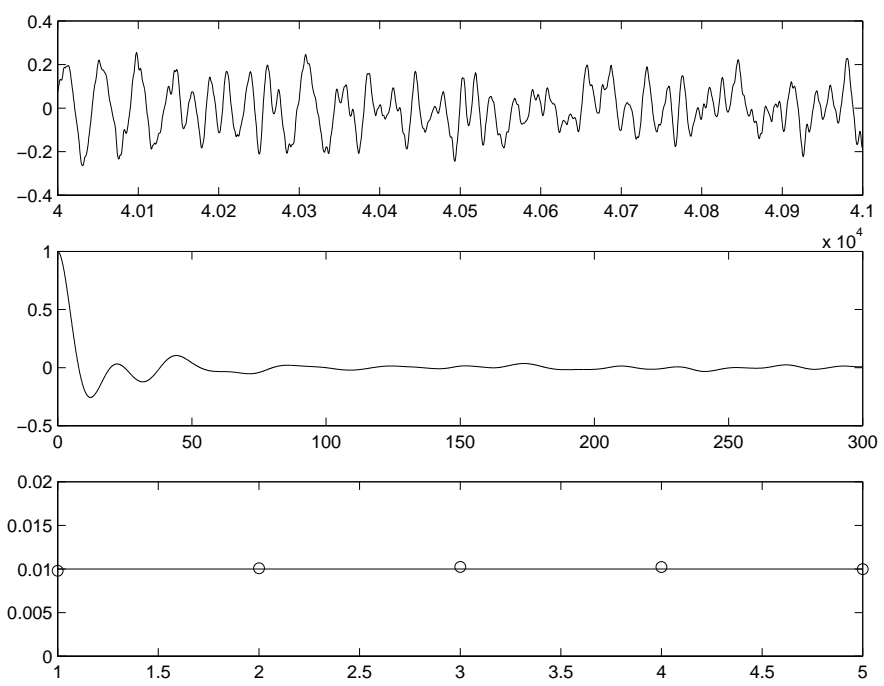

Figure 4.9: Simulations with $N=5$; Top - Time Series of Re $\hat{u}_{1}$, Middle Correlation function of Re $\hat{u}_{1}$, Bottom - Averaged Spectrum.

and random phases. Similar to the simulations discussed in [19] the initial averaging value, $T_{0}=50$, and averaging window $T=5000$ were utilized. With the time step $\Delta t=2.5 \times 10^{-4}$ the total energy in (3.6) was conserved up to $10^{-6}$.

The numerical solution exhibits three phases - the first phase, $0<$ $t<1$, is dominated by integrable behavior. Almost all of the energy is contained in Fourier modes with $k=20$ and $k=40$ and Fourier coefficients $\hat{u}_{20}$ and $\hat{u}_{40}$ follow the integrable trajectory, with the periodic exchange of energy between the two modes, while the energy is also slowly leaked into the other modes. In the second phase the solution departs from the integrable behavior and starts exhibiting chaotic, mixing properties. The solution departs from the integrable trajectory rather fast after $0.1 \%$ of energy has been transferred into the modes with $k \neq 20,40$ at time $t \approx 1.1$. Fourier modes with $k=20,40$ dominate the dynamics of the second phase when energy is redistributed between all the modes with $k \leq \Lambda$. Figures 4.10 and 4.11 illustrate the transition to chaos. Snapshots of the numerical solution, evolution of selected Fourier coefficients in time, and evolution of the energy in modes with $k=20$ and 40 are depicted in Figures 4.10 and 4.11. In the first snapshot at time $t=0$ only two frequencies are 
present in the solution. As solution evolves in time, Fourier coefficients with $k \neq 20,40$ grow in amplitude and at time $t=1.12$ more than two frequencies are present, but the spatial structure is still dominated by the modes with $k=20,40$. Next, we observe a very fast transition to chaos when the numerical solution departs quickly form the integrable trajectory. The numerical solution at time $t=1.66$ depicted in the third snapshot in Figure 4.10 shows that the solution becomes more chaotic over a very short time interval, $1.12 \leq t \leq 1.66$. Finally, in the third phase, after the time $t=20$ the instantaneous spectrum looks completely random and the dynamics resembles simulation with random initial data as reported earlier. The graphs of the absolute values of the complex Fourier modes, $k=1,20$, 40,45 , and the energy in modes with $k=20,40$ in Figure 4.11 confirm the tendency in the first, second and third phases.

Similar to other simulations with $\beta=50, \Lambda=50$ the averaged energy spectrum agrees with the predictions of the equilibrium statistical mechanics and correlation functions exhibit the same behavior as in the simulations described earlier in this paper or in [19].
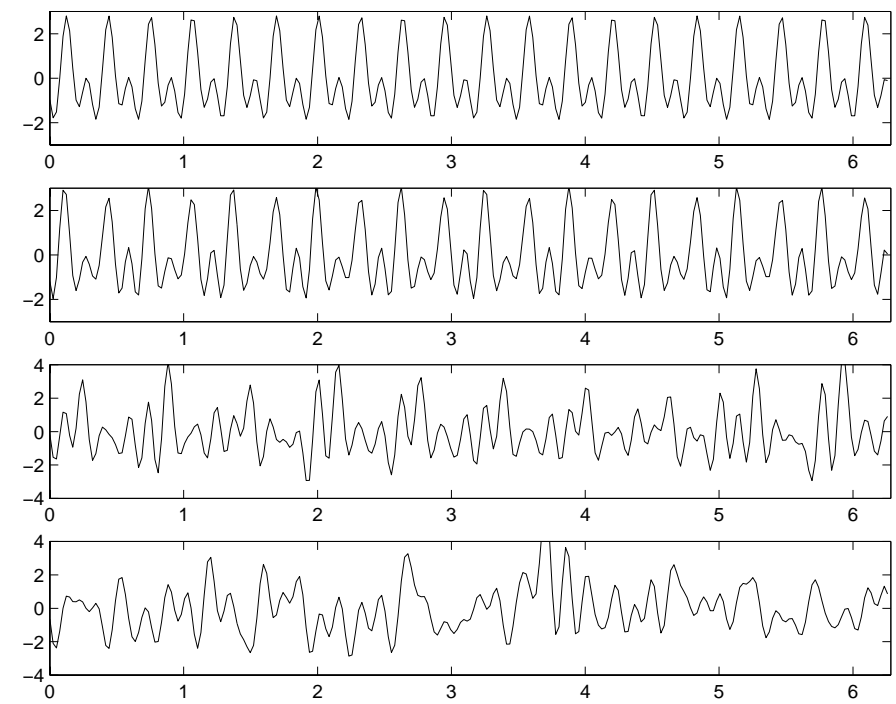

Figure 4.10: Perturbation of the $2 \mathrm{D}$ invariant subspace; Solution $u(x, t)$ at times $t=0,1.12,1.66,20$. 


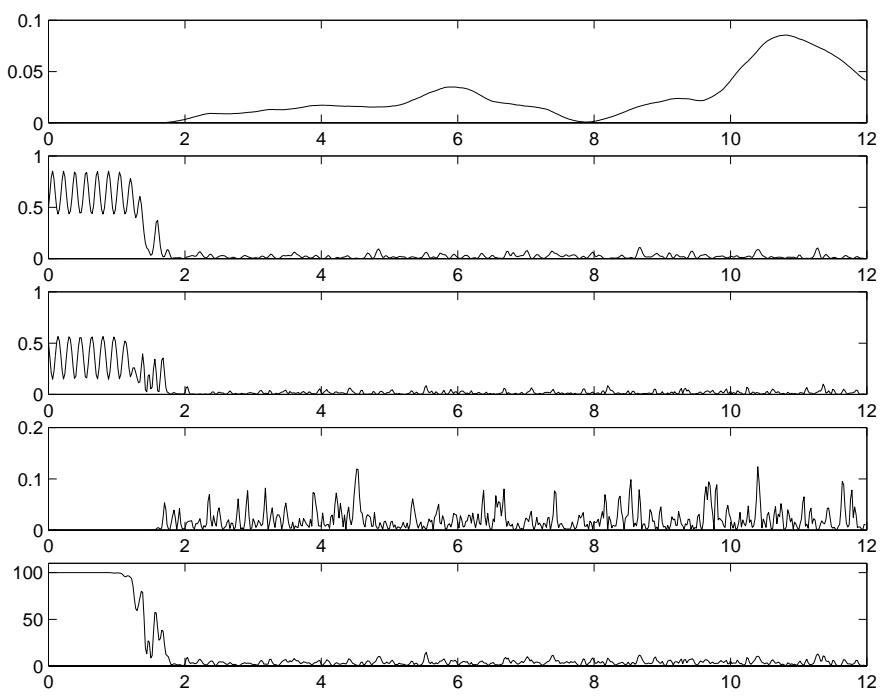

Figure 4.11: Perturbation of the 2D invariant subspace; Time evolution of the absolute values of the Fourier coefficients with $k=1$, 20, 40, 45; Bottom Energy in Modes 20 and 40 in Time.

\section{Finite-Difference Schemes with Momentum and Energy Conservation for the Burgers-Hopf Equation}

We are interested in building finite-difference schemes for the equation in (1.1) in periodic geometry which conserve a discrete form of momentum

$$
\int u d x
$$

as well as a discrete form of energy

$$
\frac{1}{2} \int u^{2} d x
$$

It is also amusing to consider schemes that might conserve a discrete form of higher powers

$$
\int u^{2 p} d x
$$

which are also conserved quantities for the equation in (1.1). 
We are interested in these properties in order to apply methods of equilibrium statistical mechanics to ensembles of these solutions as simple models. For statistical mechanics applications it is also necessary to check the Liouville property discussed in Section 2.

Below we discuss semi-discrete difference approximations to (1.1) that satisfy

(1) Discrete Conservation of Momentum

(2) Discrete Conservation of Energy

(3) Liouville property

With the properties in (5.4), as discussed in Section 2, we can always write down a Gibbs ensemble of equilibrium statistical mechanics for the difference schemes in physical space as we discuss at the end of this section.

\subsection{A Simple Five-Point Difference Scheme with Momentum and Energy Conservation}

Consider the semi-discrete five-point difference scheme

$$
\frac{d u_{j}}{d t}+\frac{1}{2} \frac{u_{j+1} u_{j+2}-u_{j-1} u_{j-2}}{3 \Delta x}=0 .
$$

Note that (5.5) is a conservation form difference approximation to (1.1). With the flux functions, $F_{j}$, consistent with (1.1) defined by

$$
F_{j}=\frac{1}{2} u_{j+\frac{1}{2}} u_{j-\frac{1}{2}}
$$

the scheme in (5.5) can be rewritten as

$$
\frac{d u_{j}}{d t}+\frac{F_{j+\frac{3}{2}}-F_{j-\frac{3}{2}}}{3 \Delta x}=0 .
$$

From the conservation form in (5.7), it follows automatically that the discrete form of momentum

$$
\sum_{j} u_{j}
$$

is conserved in time.

The discrete energy is also conserved in time by (5.5). To see this, we compute from (5.5) that

$$
\frac{d}{d t} \frac{1}{2} u_{j}^{2}+\frac{1}{2} \frac{u_{j+1} u_{j+2} u_{j}-u_{j-1} u_{j-2} u_{j}}{3 \Delta x}=0 .
$$

Thus, we introduce the nonlinear flux functions

$$
G_{j}=\frac{1}{3} u_{j-1} u_{j} u_{j+1}
$$


and rewrite the equation in (5.9) using the nonlinear flux functions, $G_{j}$, as follows

$$
\frac{d}{d t} \frac{1}{2} u_{j}^{2}+\frac{G_{j+1}-G_{j-1}}{2 \Delta x}=0
$$

so that the discrete energy,

$$
E=\frac{1}{2} \sum_{j} u_{j}^{2}
$$

is conserved in time.

The difference scheme in (5.5) also satisfies the Liouville property; this is trivial for (5.5) since

$$
\frac{\partial F_{j}}{\partial u_{j}} \equiv 0
$$

\subsection{The Goodman-Lax Finite-Difference Scheme}

The dispersive difference scheme studied by Goodman and Lax in [12] is given by

$$
\frac{d u_{j}}{d t}+u_{j} \frac{u_{j+1}-u_{j-1}}{2 \Delta x}=0 .
$$

Note that if the same nonlinear flux from (5.6) is introduced, i.e.

$$
F_{j}=\frac{1}{2} u_{j+\frac{1}{2}} u_{j-\frac{1}{2}}
$$

then the scheme in (5.14) can be written in conservation form

$$
\frac{d u_{j}}{d t}+\frac{F_{j+\frac{1}{2}}-F_{j-\frac{1}{2}}}{\Delta x}=0
$$

so that the scheme in (5.14) conserves the discrete momentum

$$
\sum_{j} u_{j}
$$

For $u_{j}>0$ Goodman and Lax observed that (5.14) can be rewritten as

$$
\frac{d}{d t} \ln u_{j}+\frac{u_{j+1}-u_{j-1}}{2 \Delta x}=0
$$

from which it is follows that

$$
\sum_{j} \ln u_{j}=\ln \left(\prod_{j} u_{j}\right)
$$

is conserved in time for $u_{j}>0$. Thus, for initial data in the cone with $u_{j}^{0}>0$, the solution stays positive for the ordinary differential equations 
in (5.14) and exists for all time $t>0$. Furthermore, Goodman and Lax showed that

On the cone of $u_{j}>0,(5.14)$ is a completely integrable system of equations which is equivalent to the Toda lattice.

\section{Explicit Examples of Nonlinear Instability for the Goodman-Lax Scheme with Initial Data Changing Sign}

In contrast to (5.20), next, explicit examples are presented which show that the ordinary differential equations in (5.14) have many oscillatory solutions which blow up in finite time when the initial data change sign.

The equation in (5.14) has the property that it propagates zeros in the initial data, i.e. if $u_{k}^{0}$ is zero, then

$$
u_{k}(t) \equiv 0
$$

for all time for the solution of (5.14). The property in (5.21) is obvious because the $k$-th equation in (5.14) has the form, $\frac{d}{d t} u_{k}=u_{k} g_{k}(\vec{u})$. To build explicit examples of solutions with finite time blow-up, we consider the solution of (5.14) with the initial data

$$
u_{j}^{0} \text { with } u_{3 k}^{0}=0 \quad \text { for all } k
$$

so that $u_{3 k}(t) \equiv 0$ by (5.21). Consider the pair of points $u_{3 k+1}(t), u_{3 k+2}(t)$ between the consecutive zero states, $u_{3 k}(t)=u_{3 k+3}(t) \equiv 0$. For simplicity in notation, denote $u_{3 k+1}(t), u_{3 k+2}(t)$ by $a_{1}(t), a_{2}(t)$, respectively. Then with (5.22) it is a simple matter to calculate that there are explicit solutions of (5.14) defined through the coupled ordinary differential equations

$$
\begin{gathered}
\frac{d a_{1}}{d t}+\frac{a_{1} a_{2}}{2 \Delta x}=0 \\
\frac{d a_{2}}{d t}-\frac{a_{1} a_{2}}{2 \Delta x}=0 \\
a_{1}(t=0)=u_{3 k+1}^{0}, a_{2}(t=0)=u_{3 k+2}^{0}
\end{gathered}
$$

with $u_{3 k}(t) \equiv 0, u_{3 k+1}(t)=a_{1}(t), u_{3 k+2}(t)=a_{2}(t)$. The equations in (5.23) trivially have the conserved quantity

$$
a_{1}(t)+a_{2}(t)=A_{k}
$$


with $A_{k}=u_{3 k+1}^{0}+u_{3 k+2}^{0}$ so that (5.24) reduces to the elementary ordinary differential equation

$$
\frac{d a_{1}}{d t}=\frac{a_{1}^{2}}{2 \Delta x}-\frac{A_{k} a_{1}}{2 \Delta x} .
$$

The ordinary differential equation in (5.25) has many solutions that blowup in finite time. In particular, for initial data that change sign so that $A_{k}=0$, we have the explicit formula for the solution of (5.14)

$$
\begin{gathered}
u_{3 k}(t)=0, \quad u_{3 k_{2}}(t)=-u_{3 k_{1}}(t) \\
u_{3 k_{1}}(t)=u_{3 k_{1}}^{0}\left(1-\frac{u_{3 k_{1}}^{0} t}{2 \Delta x}\right)^{-1}
\end{gathered}
$$

Thus, there is blow-up in finite time of order $O(\Delta x)$, for any initial data for (5.14) consistent with (5.25) so that $u_{3 k_{1}}^{0}>0$ for some $k$ and with $u_{3 k_{2}}^{0}=-u_{3 k_{1}}^{0}$. In particular,

\section{The difference scheme in (5.14) never conserves} any discrete power $\sum u_{j}^{2 p}$ for all initial data.

Thus, the conservation properties of the five-point difference scheme in (5.5) strongly contrast with these for the difference scheme in (5.14) even though both schemes are based on the same nonlinear flux from (5.6) and (5.15) with the flux evaluated at slightly different points for (5.5) than for (5.14). However, it is worth remarking here that the difference scheme in (5.14) satisfies the Liouville property since

$$
\frac{\partial F_{j}}{\partial u_{j}}=-\frac{u_{j+1}-u_{j-1}}{2 \Delta x}
$$

so that

$$
\sum_{j} \frac{\partial F_{j}}{\partial u_{j}}=0
$$

Next, we briefly consider the nonlinear difference scheme

$$
\frac{d u_{j}}{d t}+\frac{1}{2} \frac{u_{j+1}^{2}-u_{j-1}^{2}}{2 \Delta x}=0
$$

which obviously has conservation form with the flux

$$
F_{j}=\frac{1}{2} u_{j}^{2} .
$$

Note that this scheme is also structurally close to the scheme in (5.5). This scheme also has exact solutions with an ansatz satisfying a restricted 
version of the symmetries in the first line of (5.26) which exhibit nonlinear instability. Mimicing (5.26), define exact solutions of (5.29) with the form

$$
u_{3 k}(t)=0, \quad u_{3 k+1}(t)=a_{1}(t), \quad u_{3 k+2}(t)=-a_{1}(t)
$$

By substituting the ansatz from (5.31) into (5.29) these functions define an exact solution provided $a_{1}(t)$ satisfies the nonlinear ordinary differential equation

$$
\frac{d a_{1}}{d t}=-\frac{a_{1}^{2}}{4 \Delta x}, \quad a_{1}(0)=u_{1}^{0} .
$$

Note that this ordinary differential equation for $a_{1}$ in (5.32) has a coefficients with one-half the magnitude and the opposite sign of (5.25) with $A_{k}=0$. In particular, the explicit solutions of (5.29) exhibit nonlinear instability and finite time blow-up provided that $u_{1}^{0}$ satisfies $u_{1}^{0}<0$.

Next, we consider the composite difference scheme combining (5.14) and (5.29) with the form

$$
\frac{d u_{j}}{d t}+\alpha \frac{u_{j}\left(u_{j+1}-u_{j-1}\right)}{2 \Delta x}+(1-\alpha) \frac{1}{2} \frac{u_{j+1}^{2}-u_{j-1}^{2}}{2 \Delta x}=0
$$

where $0 \leq \alpha \leq 1$ is a parameter. All of these schemes are consistent with the Burgers-Hopf equation in (1.1). They admit exact solutions with the form in (5.31) provided $a_{1}(t)$ satisfies the ordinary differential equation

$$
\frac{d a_{1}}{d t}=\left(\frac{\alpha}{2 \Delta x}-\frac{1-\alpha}{4 \Delta x}\right) a_{1}^{2}, \quad a_{1}(0)=u_{1}^{0} .
$$

\subsection{Another Finite-Difference Scheme with Momentum and Energy Conservation}

We ask whether there is a specific value of $\alpha$ for the composite scheme in (5.33) so that there are no elementary exact solutions with the structure in (5.31) exhibiting nonlinear instability through finite time blow-up. From (5.34), we see that the choice $\alpha=1 / 3$ achieves this. For this value of $\alpha$ the scheme in (5.33) becomes

$$
\frac{d u_{j}}{d t}+\frac{1}{3}\left(u_{j+1}+u_{j}+u_{j-1}\right) \frac{u_{j+1}-u_{j-1}}{2 \Delta x}=0 .
$$

This is the scheme introduced by Kruskal and Zabusky [14] in their pioneering numerical studies on soliton interactions. Two phase oscillations in this scheme have also been studied recently by Levermore and Liu, [20]. Not only does this difference scheme eliminate nonlinear instability, Kruskal 
and Zabusky have observed that it conserves both the momentum and the energy. In fact the scheme has two local conservation forms

$$
\begin{aligned}
\frac{d u_{j}}{d t}+\frac{F_{j+\frac{1}{2}}-F_{j-\frac{1}{2}}}{\Delta x} & =0 \\
\frac{1}{2} \frac{d u_{j}^{2}}{d t}+\frac{G_{j+\frac{1}{2}}-G_{j-\frac{1}{2}}}{\Delta x} & =0
\end{aligned}
$$

with

$$
\begin{gathered}
F_{j+\frac{1}{2}}=\frac{1}{6}\left(u_{j}^{2}+u_{j} u_{j+1} u_{j+1}^{2}\right) \\
G_{j+\frac{1}{2}}=\frac{1}{6}\left(u_{j}^{2} u_{j+1}+u_{j} u_{j+1}^{2}\right) .
\end{gathered}
$$

The scheme in (5.35) also satisfies the Liouville property since

$$
\frac{\partial F_{j}}{\partial u_{j}}=-\frac{1}{3} \frac{u_{j+1}-u_{j-1}}{2 \Delta x}
$$

so that

$$
\operatorname{div} \vec{F}=\sum_{j} \frac{\partial F_{j}}{\partial u_{j}}=0
$$

An Important Open Problem for Testing Statistical Theories Are there elementary difference schemes which have the Liouville property and conserve, in addition, the 4-th moment

$$
\sum_{j} u_{j}^{4}
$$

(or some approximation to this quantity) besides the momentum and the energy? A difference scheme with these properties can be utilized to test the role of the higher order conserved quantities in the statistical theory for the simplest one-dimensional example involving the Burgers dynamics.

\subsection{Statistical Mechanics for the Difference Schemes}

Here we consider difference approximations for the Burgers-Hopf equation, $u_{t}+u u_{x}=0$, that have the three properties discussed in (5.4). For concreteness, we consider the difference scheme in (5.5) which, as we established earlier, has all three properties in (5.4). We consider periodic solutions for simplicity on the normalized period interval $[0,2 \pi]$ and assume that there are $2 N+1$ discrete distinct points with $(2 N+1) \Delta x=2 \pi, u_{j}(t)=u(j \Delta x, t)$, and $\vec{u}=\left(u_{0}, u_{1}, \ldots, u_{2 N}\right)$. Periodicity requires

$$
u_{j+2 N+1}=u_{j} .
$$


Associated with this discrete set of points is the normalized discrete inner product

$$
(\vec{f}, \vec{g})_{\Delta x}=\frac{\Delta x}{2 \pi} \sum_{j=0}^{2 N} f_{j} g_{j}^{*}=\frac{\Delta x}{2 \pi} \vec{f} \cdot \vec{g}
$$

where $\vec{f} \cdot \vec{g}$ is the standard complex inner product on $C^{2 N+1}$ with $(\vec{f}, \vec{f})_{\Delta x}=$ $|\vec{f}|_{\Delta x}^{2}$.

The momentum constraint,

$$
\sum_{j=0}^{2 N} u_{j}=\text { Const }
$$

is a rugged constraint satisfied for all solutions and does not have a statistical character. For simplicity, below we consider solutions with zero total momentum. In contrast, fluctuations in the energy are statistical. As a consequence of the Liouville property any function of the energy is an invariant measure for the dynamics. However, the most probable invariant measure for the dynamics given an average total energy and zero total momentum is the Gibbs measure with $d P_{\beta}(\vec{u})$ given by

$$
d P_{\beta}(\vec{u})=C_{\beta} \operatorname{EXP}\left(-\frac{\beta}{2}|\vec{u}|_{\Delta x}^{2}\right) \prod_{j=0}^{2 N} d u_{j} \times \delta\left(\sum_{j=0}^{2 N} u_{j}\right)
$$

Our interest in the statistical mechanics of these systems is motivated by the fact that they should exhibit a wide range of scaling behaviors of correlations with the largest spatial scales having the longest correlations. The easiest way to check this behavior is to utilize discrete Fourier series and look at the correlation behavior for fixed spatial Fourier modes in phase space. It is also interesting to compare the behavior of correlation functions at large scales for the statistical dynamics of difference approximations as discussed in Section 5 to those for the Fourier Galerkin truncated Burgers dynamics which is naturally defined in phase space. Is the behavior universal at large scales? Not surprisingly, in the next section, we utilize discrete Fourier series to establish connection between the two methods. 


\section{Physical Space and Fourier Phase Space Representations of Difference Schemes and Statistical Mechanics}

We begin this section with a summary of well-known properties of discrete Fourier series.

\subsection{Summary of Discrete Fourier Series}

Given any $2 \pi$-periodic function, $f(x)$, we associate with $f(x)$, the discrete vector $\vec{f}$ via

$$
f(x) \rightarrow[f(0), f(\Delta x), f(2 \Delta x), \ldots, f(2 N \Delta x)]=\vec{f}
$$

with

$$
(2 N+1) \Delta x=2 \pi .
$$

The trigonometric functions $e^{i k x}=e_{k}(x)$ for $k=0, \pm 1, \pm 2, \ldots$ are a complete orthonormal basis with the continuous inner product

$$
(f, g)=\frac{1}{2 \pi} \int_{0}^{2 \pi} f g^{*} d x
$$

so that

$$
\left(e_{k}(x), e_{j}(x)\right)=\delta_{k, j},
$$

where $\delta_{k, j}$ is the Kronecker delta. The discrete functions,

$$
\vec{e}_{k}=\left(1, e^{i k \Delta x}, e^{i 2 k \Delta x}, \ldots, e^{i 2 N k \Delta x}\right)
$$

for $|k| \leq N$ are also an orthonormal basis for $C^{2 N+1}$ so that

$$
\left(\vec{e}_{k}, \vec{e}_{j}\right)_{\Delta x}=\delta_{k, j} \quad \text { for }|k|,|j| \leq N .
$$

For every $\vec{f} \in C^{2 N+1}$, the discrete Fourier coefficients are given by

$$
\hat{f}_{k}^{\Delta x}=\left(\vec{f}, \vec{e}_{k}\right)_{\Delta x} \quad \text { for }|k| \leq N
$$

and every $\vec{f}$ admits the unique expansion

$$
\vec{f}=\sum_{|k| \leq N} \hat{f}_{k}^{\Delta x} \vec{e}_{k}, \quad(2 N+1) \Delta x=2 \pi
$$

with

$$
|\vec{f}|_{\Delta x}^{2}=\sum_{|k| \leq N}\left|\hat{f}_{k}^{\Delta x}\right|^{2}
$$


For real-valued discrete functions, we necessarily have

$$
\left(\hat{f}_{k}^{\Delta x}\right)^{*}=\hat{f}_{-k}^{\Delta x} \quad \text { for }|k| \leq N .
$$

Thus, it follows from (6.6), (6.7) and (6.8) that for real-valued functions, the linear map from $R^{2 N+1}$ to $R^{2 N+1}$ defined by discrete Fourier transform

$$
\mathrm{FT}: \vec{f} \rightarrow\left[\hat{f}_{0}^{\Delta x}, \hat{f}_{1}^{\Delta x}, \hat{f}_{2}^{\Delta x}, \ldots, \hat{f}_{N}^{\Delta x}\right]
$$

is an invertible map. This map is an isometry with a suitable norm on $R^{2 N+1}$ since

$$
|\vec{f}|_{\Delta x}^{2}=\left|\hat{f}_{0}^{\Delta x}\right|^{2}+2 \sum_{k=1}^{N}\left|\hat{f}_{k}^{\Delta x}\right|^{2} .
$$

Finally, given a discrete function, $\vec{f}$, we can build the continuous trigonometric polynomial interpolant for this function by extending (6.6) so that we have the map

$$
\vec{f} \rightarrow \sum_{|k| \leq N} \hat{f}_{k}^{\Delta x} e^{i k x}
$$

For this smooth interpolant, clearly $f_{k}^{\Delta x}=f_{k}$ where $f_{k}$ is the continuous Fourier coefficient. With these preliminaries, we are finally able to discuss the relationship between equilibrium statistical mechanics in physical space and Fourier space.

\subsection{The Equivalence of Canonical Measures on Physical Space and Fourier Space}

The canonical Gibbs measure in (5.41) is a measure in physical space. It is interesting to see the form of this measure in phase space by utilizing the discrete Fourier representation.

Recall from (6.9) and (6.7) that the map, $\mathrm{FT}(\vec{u})$, is a linear map from $R^{2 N+1}$ to $R^{2 N+1}$. Next, recall the elementary fact: any map such as $\operatorname{FT}(\vec{u})$ from one measure space to another (in this particular case from $R^{2 N+1}$ to $R^{2 N+1}$ ) induces a corresponding push-forward mapping on probability measures via the formula

$$
\int_{R^{2 N+1}} \Phi\left(\hat{u}_{0}^{\Delta x}, \hat{u}_{1}^{\Delta x}, \ldots, \hat{u}_{N}^{\Delta x}\right) d \mathrm{FT}(v)=\int_{R^{2 N+1}} \Phi(\mathrm{FT}(\vec{u})) d v
$$

for all bounded continuous functions $\Phi$. In (6.12) $d v$ is the physical space probability measure on $R^{2 N+1}$ and $d \mathrm{FT}(v)$ denotes the induced probability measure on phase space, $R^{2 N+1}$. 
Proposition 6.1. The probability measure induced by the Gibbs measure in (5.41) under the discrete Fourier series mapping FT is given by

$$
d \operatorname{FTP} P_{\beta}\left(\hat{u}_{0}^{\Delta x}, \hat{u}_{1}^{\Delta x}, \ldots, \hat{u}_{N}^{\Delta x}\right)=C_{\beta} \operatorname{EXP}\left(-\beta \sum_{k=1}^{N}\left|\hat{u}_{k}^{\Delta x}\right|^{2}\right) \times \delta\left(\hat{u}_{0}^{\Delta x}\right) .
$$

Thus, the canonical measure in phase space utilized for studying the statistical mechanics of spectrally truncated Burgers dynamics (TBD) is precisely the measure induced on phase space by the canonical Gibbs measure from (5.41) in physical space associated with various finite-difference approximations. This result links the two approaches and demonstrates their equivalence with regard to stationary measures.

The proof of the proposition is straightforward. First, from (6.10) the push-forward of the function, $\operatorname{EXP}\left(-\beta|\vec{f}|_{\Delta x}^{2}\right)$ is given by

$$
\operatorname{EXP}\left[-\beta\left(\left|\hat{f}_{0}^{\Delta x}\right|^{2}+2 \sum_{k=1}^{N}\left|\hat{f}_{k}^{\Delta x}\right|^{2}\right)\right] .
$$

Second, the push-forward of $\delta\left(\sum_{j=0}^{2 N} u_{j}\right)$ is $\tilde{C} \delta\left(\hat{u}_{0}\right)$. With these two facts, it is easy for the reader to complete the proof.

\subsection{Finite-Difference Dynamics as Fourier Space Dynamics}

We write the finite difference equation in (5.5),

$$
\frac{d u_{j}}{d t}+\frac{1}{2} \frac{u_{j+1} u_{j+2}-u_{j-1} u_{j-2}}{3 \Delta x}=0
$$

equivalently as coupled system of ordinary differential equations in Fourier space so that this discrete method can be compared to the Galerkin truncated dynamics discussed in Section 3. We use the interpolated Fourier series

$$
u^{\Delta x}(x, t)=\sum_{|k| \leq N} \hat{u}_{k}^{\Delta x} e^{i k x}
$$

to simplify calculations. We suppress the $\Delta x$ superscript below for simplicity. Recall that the equations in (6.14) can be rewritten in conservation form

with the flux function

$$
\frac{d u_{j}}{d t}+\frac{F_{j+\frac{3}{2}}-F_{j-\frac{3}{2}}}{3 \Delta x}=0
$$

$$
F_{j}=\frac{1}{2} u_{j+\frac{1}{2}} u_{j-\frac{1}{2}} .
$$


First, we write the Fourier series expansions for $u_{j+\frac{1}{2}}$ and $u_{j-\frac{1}{2}}$

$$
\begin{aligned}
& u_{j+\frac{1}{2}}=\sum_{|k| \leq N} \hat{u}_{k} e^{i \frac{k \Delta x}{2}} e^{i k x} \\
& u_{j-\frac{1}{2}}=\sum_{|l| \leq N} \hat{u}_{l} e^{-i \frac{l \Delta x}{2}} e^{i l x}
\end{aligned}
$$

and compute the Fourier expansion for the flux function

$$
F_{j}=\frac{1}{2} u_{j+\frac{1}{2}} u_{j-\frac{1}{2}}=\sum_{|m|,|l| \leq N} \hat{u}_{m} \hat{u}_{l} e^{i \frac{(m-l) \Delta x}{2}} e^{i(m+l) x}
$$

and

$$
\begin{aligned}
& \frac{F_{j+\frac{3}{2}}-F_{j-\frac{3}{2}}}{3 \Delta x}= \\
& \quad=\frac{1}{6 \Delta x} \sum_{|m|,|l| \leq N}\left(e^{i(2 m+l) \Delta x}-e^{-i(2 l+m) \Delta x}\right) \hat{u}_{m} \hat{u}_{l} e^{i(m+l) x} .
\end{aligned}
$$

Thus, we introduce the symbol

$$
D_{m, l}=\frac{1}{6 \Delta x}\left(e^{i(2 m+l) \Delta x}-e^{-i(2 l+m) \Delta x}\right)
$$

which is an analog of $x$-differentiation, so that

$$
\frac{F_{j+\frac{3}{2}}-F_{j-\frac{3}{2}}}{3 \Delta x}=\sum_{|m|,|l| \leq N} D_{m, l} \hat{u}_{m} \hat{u}_{l} e^{i(m+l) x} .
$$

Next, we need to take into account aliasing since the right hand side for (6.14) is evaluated at the mesh points, $j \Delta x$, i.e.

$$
e^{i k x}=e^{i(k+p(2 N+1)) x}, \quad \text { for } x=j \Delta x
$$

and $(2 N+1) \Delta x=2 \pi$. For a fixed $k$ with $|k| \leq N$, we need to consider the contribution from the points

$$
m+l=k \pm(2 N+1), \quad 0<|m| \leq N, 0<|l| \leq N .
$$

The contribution to aliasing is from the modes with

$$
\begin{array}{rlrl}
m+l & =k-(2 N+1), & & k>0 \\
m+l=k+(2 N+1), & & k<0 .
\end{array}
$$


Thus, in Fourier space utilizing the discrete Galerkin projection we get the equivalent dynamics for the difference approximation in (6.14)

$$
\begin{aligned}
\frac{d}{d t} \hat{u}_{k} & =\sum_{m+l=k} D_{m, l} \hat{u}_{m} \hat{u}_{l}+ \\
& \sum_{m+l=k-2 N-1 ; k>0} D_{m, l} \hat{u}_{m} \hat{u}_{l}+ \\
& \sum_{m+l=k+2 N+1 ; k<0} D_{m, l} \hat{u}_{m} \hat{u}_{l}, \quad 0<|k| \leq N .
\end{aligned}
$$

We rewrite equations in (6.25) through the complex conjugates, $\hat{u}_{m}^{*}$, $\hat{u}_{l}^{*}$ using the property $D_{-m,-l}=D_{l, m}$

$$
\begin{aligned}
& \frac{d}{d t} \hat{u}_{k}=\sum_{k+m+l=0} D_{l, m} \hat{u}_{m}^{*} \hat{u}_{l}^{*}+ \\
& \quad \sum_{k+m+l=2 N+1 ; k>0} D_{l, m} \hat{u}_{m}^{*} \hat{u}_{l}^{*}+ \\
& \quad \sum_{k+m+l=-2 N-1 ; k<0} D_{l, m} \hat{u}_{m}^{*} \hat{u}_{l}^{*}, \quad 0<|k| \leq N .
\end{aligned}
$$

Also we recall that the equations for the Galerkin spectral truncation can be written in the following (alternative to (3.4)) form

$$
\frac{d}{d t} \hat{u}_{k}=-\sum_{k+m+l=0}-i m \hat{u}_{m}^{*} \hat{u}_{l}^{*}=\sum_{k+m+l=0} \frac{i(m+l)}{2} \hat{u}_{m}^{*} \hat{u}_{l}^{*}
$$

where $i(m+l) / 2=-i k / 2$ corresponds to the operator of differentiation $\partial / \partial x$ in physical space. Next, we consider $D_{l, m}$ and demonstrate that $D_{l, m}$ is also a finite-difference approximation to the operator of differentiation $\partial / \partial x$

$$
\begin{gathered}
D_{l, m}=\frac{1}{6 \Delta x}\left[e^{i(2 l+m) \Delta x}-e^{-i(2 m+l) \Delta x}\right]= \\
\left.\left.\frac{1}{6 \Delta x}[\cos (2 l+m) \Delta x)-\cos (2 m+l) \Delta x\right)\right]+ \\
\frac{i}{3 \Delta x} \sin \left(\frac{3(m+l)}{2} \Delta x\right) \cos \left(\frac{m-l}{2} \Delta x\right) .
\end{gathered}
$$

We can disregard the first term, $\cos (2 l+m) \Delta x)-\cos (2 m+l) \Delta x)$, in (6.28) because of the symmetry between the two coefficients, $m$ and $l$, entering in 
(6.26). Thus,

$$
\lim _{\Delta x \rightarrow 0} D_{l, m}=\frac{i(m+l)}{2} .
$$

Similarly, we can show that the finite-difference scheme in (5.35) first introduced by Kruskal and Zabusky is equivalent to the system of ordinary differential equations in (6.26) for the discrete Fourier coefficients with a different approximation, $D_{m, l}^{\prime}$, for the space derivative $\partial / \partial x$

$$
D_{m, l}^{\prime}=\frac{i}{3 \Delta x}\left[\sin ((l+m) \Delta x)+\sin \left(\frac{(l+m) \Delta x}{2}\right) \cos \left(\frac{(m-l) \Delta x}{2}\right)\right]
$$

with the same limit as $\Delta x \rightarrow 0$, i.e.

$$
\lim _{\Delta x \rightarrow 0} D_{l, m}^{\prime}=\frac{i(m+l)}{2} .
$$

\subsection{Comparison of the Galerkin Spectral Truncation and Finite-Difference Schemes and the Role of Aliasing terms}

We consider equations in (6.26) in more detail and show that the aliasing terms might play an important role in the finite-difference approximations.

Explicit Formulas for the Low-Dimensional Case $N=1$ First, we consider the simplest situation with $N=1$, so that $2 N+1=3$. In this case the only wavenumbers contributing to the equations for the Fourier coefficients in (6.26) are $m, l= \pm 1$. Therefore, the Galerkin spectral truncation trivially reduces to the equation

$$
\frac{d}{d t} \hat{u}_{1}=0 .
$$

The first term on the right-hand side of the equation in (6.26) is zero (there are no wavenumbers $m$ and $l$ satisfying $m+l= \pm 1$ ) but, the finitedifference equations in Fourier space in (6.26) are non-trivial because of the contribution of the second aliasing term since for $k=m=l=1$, the condition $k+l+m=2 N+1=3$ is satisfied. Thus, the equations in (6.26) reduce to a non-trivial ordinary differential equation for $\hat{u}_{1}$

$$
\frac{d}{d t} \hat{u}_{1}=D_{1,1}\left(\hat{u}_{1}^{*}\right)^{2}
$$

Substituting the explicit expressions for $D_{m, l}$ and $D_{m, l}^{\prime}$ we obtain that the five-point scheme in (6.14) and Kruskal-Zabusky scheme in (5.35) for 
$N=1$ are equivalent to the following ordinary differential equation for $\hat{u}_{1}$, respectively,

$$
\frac{d}{d t} \hat{u}_{1}=\frac{i}{3 \Delta x} \sin (3 \Delta x)\left(\hat{u}_{1}^{*}\right)^{2}
$$

and

$$
\frac{d}{d t} \hat{u}_{1}=\frac{i}{3 \Delta x}[\sin (2 \Delta x)+\sin (\Delta x)]\left(\hat{u}_{1}^{*}\right)^{2} .
$$

Explicit Formulas for the Wavenumber $k=1$ The equations in (6.26) for $k=1$ and general $N$ become

$$
\begin{array}{r}
\frac{d}{d t} \hat{u}_{1}=\sum_{m+l+1=0} D_{l, m} \hat{u}_{m}^{*} \hat{u}_{l}^{*}+ \\
\sum_{m+l+1=2 N+1} D_{l, m} \hat{u}_{m}^{*} \hat{u}_{l}^{*} .
\end{array}
$$

The first term in the equation in (6.36) is the usual interaction term common for the finite-difference approximation and Fourier Galerkin truncation, but the second term is the contribution from aliasing. The contribution from aliasing can be rewritten more explicitly as follows

$$
\sum_{m+l+1=2 N+1} D_{l, m} \hat{u}_{m}^{*} \hat{u}_{l}^{*}=\sum_{|l| \leq N} D_{l, 2 N-l} \hat{u}_{2 N-l}^{*} \hat{u}_{l}^{*}=D_{N, N}\left(\hat{u}_{N}^{*}\right)^{2} .
$$

To collapse the sum to a single term in (6.37), we have utilized the fact that $D_{l, m}=0$ for $|m|>N$ or $|l|>N$. It is easy for the reader to check that as $\Delta x \rightarrow 0, \lim D_{N, N} \neq 0$ for either of the two difference schemes with two different non-zero limiting values.

When the energy in the equations in (6.26) is statistically equipartitioned so that there is a significant energy at the largest wavenumbers, the contribution from aliasing in (6.37) does not decay as the spatial resolution of the finite-difference schemes is increased, i.e. $\Delta x \rightarrow 0$. Similarly we can show that any of the equations for other wavenumbers will have significant contributions from the aliasing terms when there is an equipartition spectrum. Thus, the two finite-difference schemes are actually two different models to check statistical behavior besides the Fourier Galerkin method studied earlier. 


\section{Numerical Evidence for Ergodicity and Mixing for Finite-Difference Schemes}

Here numerical evidence is presented which demonstrates that the behavior of the correlation functions for Fourier coefficients in the Fourier Galerkin truncation and in the finite-difference schemes in (5.5) and (5.35) is universal in many respects with some subtle differences. We also contrast the three discrete approximations for the Burgers-Hopf model to show quantitative difference in the statistics and illustrate the role of the aliasing terms in the finite-difference approximations.

We do not expect the behavior of either of the difference schemes to converge exactly to the behavior of the Fourier Galerkin truncation in the statistical regime with large energies at high wavenumbers. Our reason for this has already been discussed in Section 6.4; near the statistical equipartition regime, the new aliasing terms for the finite-difference schemes are not negligible for the dynamics and do not vanish as $N \rightarrow \infty$. Nevertheless, the two difference schemes provide alternative nonlinear dynamics to check universal features of the statistical predictions which we have established previously in Sections 3, 5 and 6 of the present paper and already confirmed dramatically for the Fourier Galerkin truncation. In general, we will find below that the Kruskal-Zabusky finite-difference scheme from 5.3 has an equipartition spectrum with ergodicity and mixing with time correlations that also agree with the predicted scaling theory. On the other hand, the five-point scheme described in Section 5.1 typically reproduces the equipartition spectrum except for some peculiar behavior near a resonant band of wavenumber, $k^{r} \approx(2 N+1) / 3$. In this band there are long range correlations and if $(2 N+1) / 3$ happens to be an integer, we present examples below where a steady mode can appear at this wavenumber which is not in equipartition of energy. Thus, the temporal correlation scaling theory for statistical solutions of the five-point scheme is a much worse approximation in this case and this approximation can even fail to have mixing dynamics for a single mode in a bath of modes which display strong mixing.

To compare the five-point finite-difference scheme in (5.5) and the Kruskal-Zabusky finite-difference scheme in (5.35) with the simulations with the Fourier Galerkin truncation reported in [19] we perform simulations with the deterministic initial condition $u_{0}=2 \sin (x)$. At each time step the discrete Fourier transform is utilized to compute the statistics in Fourier space. To demonstrate the mixing properties of the finite-difference schemes the time-averaging of an individual solution is performed; thus, 
analogous to the Fourier Galerkin truncation, the energy in the $k$-th mode is computed by (4.1). For the simulations with finite-difference schemes the initial averaging value, $T_{0}=1000$, and averaging window, $T=40000$, are utilized. The total energy in physical space

$$
E=\frac{\Delta x}{4 \pi} \sum_{j=1}^{2 N+1} u_{j}^{2}
$$

has the value $E=1$. For fourth-order Runge-Kutta time differencing with the time step $\Delta t=5 \times 10^{-4}$ the energy was conserved within $10^{-5}$ relative error. The value of $N$ is 50 , so that the number of the spatial points is $2 N+1=101$, and the corresponding $\beta$ is $\beta=2 E /(2 N+1)=50.5$. We would like to emphasize that in simulations for finite-difference schemes in (5.5) and (5.35) with $2 N+1$ spatial points the largest non-zero wavenumber is $N$.

Let us recall the results in [19] for simulations with the deterministic initial condition $u_{0}=2 \sin (x)$ with the pseudo-spectral scheme. In particular, in order to compare the simulations for the finite-difference schemes we reproduce the snapshots of the solution depicted in Figure 7.1, the scaling of correlation times in Figure 7.2, and also show the correlation functions for selected wavenumbers in Figure 7.3.

\subsection{Kruskal-Zabusky Finite-Difference Scheme}

Similar to the simulation with the Fourier Galerkin projection, the numerical solution of the Kruskal-Zabusky finite-difference scheme in (5.35) exhibits three phases depicted in the snapshots of the solution in Figure 7.4 at times $t=0.4,0.6,1.6,20$. Although, like in the Galerkin spectral projection, the energy is transfered into the Fourier modes with high wavenumbers, $k \approx N$, the mechanism of the shock brake-up is different for the finite-difference scheme in (5.35) compared with the Galerkin spectral truncation. The solution computed with the Kruskal-Zabusky scheme becomes oscillatory near the shock, but remains smooth almost everywhere in the interval $[0,2 \pi]$. As shown in Figure 7.1, the situation for the Galerkin spectral truncation is exactly the opposite, since after the classical brakeup time the solution in Figure 7.1 becomes highly oscillatory outside of the shock on the whole interval $[0,2 \pi]$.

During the second phase the solution becomes very oscillatory with an increase of the energy in high wavenumbers and then the energy is 

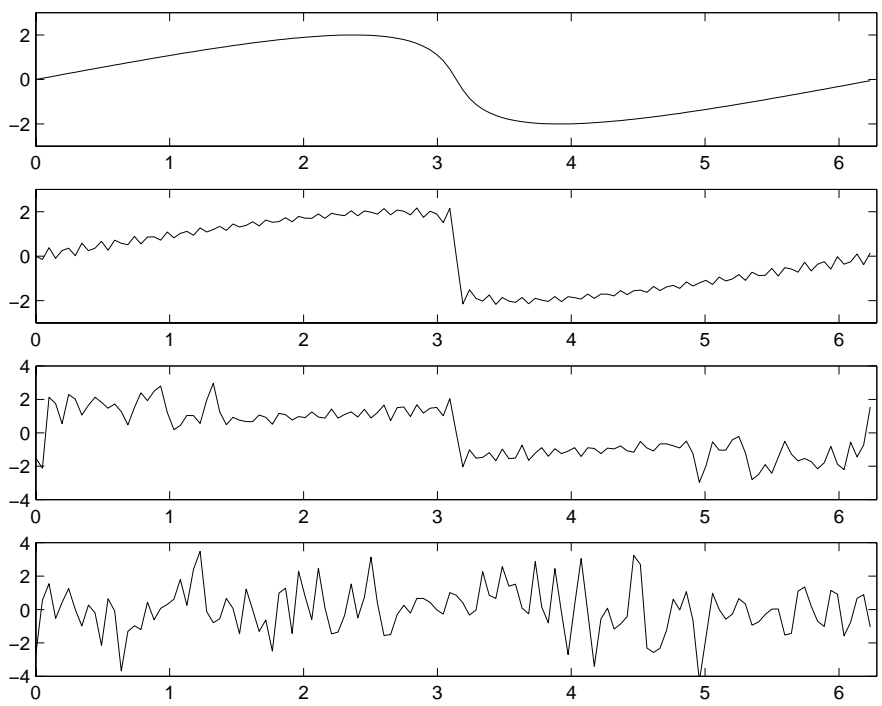

Figure 7.1: Galerkin truncation. Simulation with $\beta=50, \Lambda=50, u_{0}=2 \sin (x)$. Solution at time $t=0.4,0.56,1.56,20$.

redistributed between all the Fourier modes, and by time $t=20$ the instantaneous spectrum depicted in Figure 7.5 looks completely random. We obtain excellent agreement between the predictions of equilibrium statistical mechanics and the numerical estimates for the spectrum of the Fourier coefficients presented in Figure 7.6. Clearly, there is a statistical equipartition of energy for times $t>1000$. The relative errors are distributed uniformly in this case, and do not exceed $2 \%$.

Correlation functions for the Fourier coefficients computed in simulations with the finite-difference scheme in (5.35) (Figure 7.7) have very different structure than correlation functions computed with the Fourier Galerkin projection. In particular, the correlation functions for low wavenumbers presented in Figure 7.7 are highly oscillatory and, thus, become negative, while correlation functions computed in the simulation with the Galerkin projection (Figure 7.3) are strictly positive. For $k=1$, this feature is clearly a manifestation of the additional aliasing term from (6.37) in the dynamics. Therefore, in order to test the scaling theory for the Kruskal-Zabusky finite-difference scheme we need to consider the signed area under the curve 


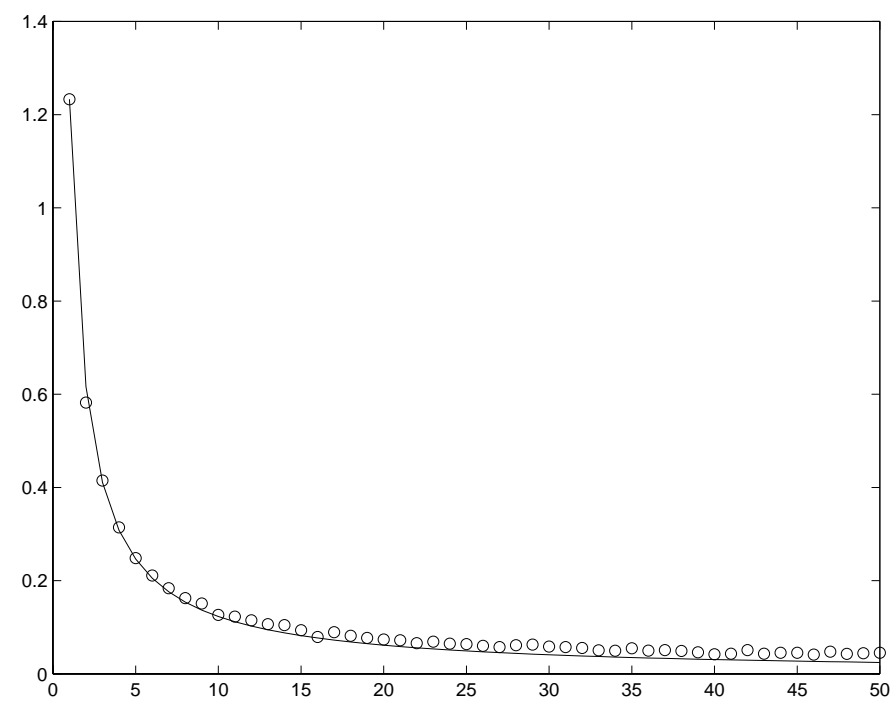

Figure 7.2: Galerkin truncation. Simulation with $\beta=50, \Lambda=50, u_{0}=2 \sin (x)$. Correlation Times; Circles - DNS, Solid Line - Predictions of the Scaling Theory.

of correlation functions. This, essentially, amounts to computing the correlation times as areas under the absolute values of correlation functions of $\operatorname{Re} \hat{u}_{k}$.

The range in time scales of correlations in the individual Fourier modes varies over 2 decades for the value $\beta=50.5$ in the present simulation. The elementary scaling theory for correlation times of the Fourier coefficients developed in (3.11) is compared with the numerically computed correlation times in Figure 7.8. Numerical results presented in Figure 7.8 demonstrate that the simple scaling theory works very well for low wavenumbers. The only qualitative difference with the Galerkin spectral projection (see Figure 7.2 ) is that the correlation times for higher wavenumbers, $k \geq 35$, increase a little bit with $k$. This might be explained as a purely numerical issue caused by utilizing the absolute value of the correlation function for computing the correlation times; in this case the errors from the unresolved tails might accumulate and cause the correlation times to increase slightly with $k$. 

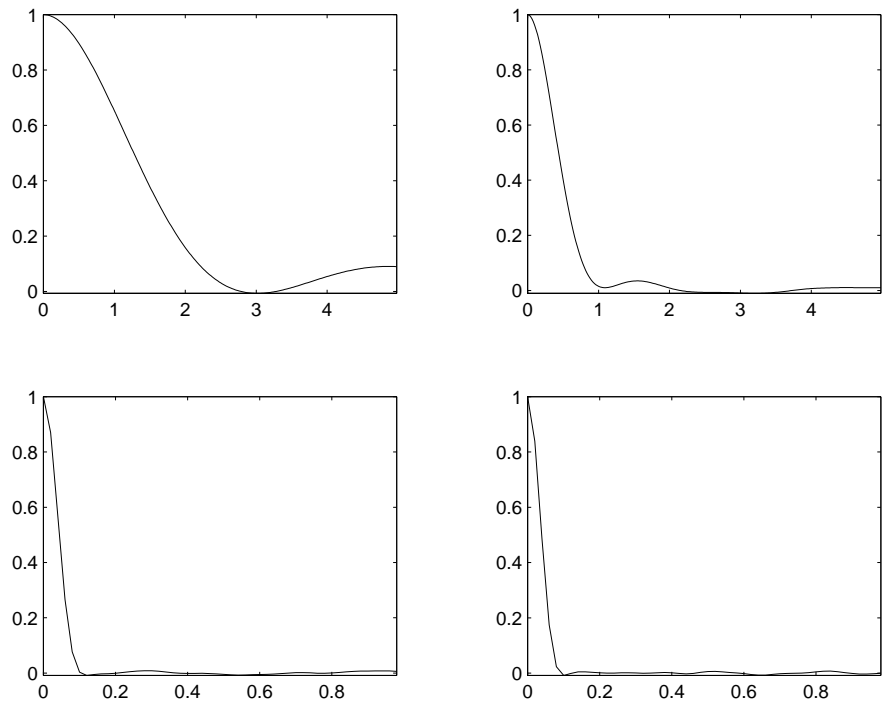

Figure 7.3: Galerkin truncation. Simulation with $\beta=50, \Lambda=50, u_{0}=2 \sin (x)$. Correlation Functions for Re $\hat{u}_{k}$ with $k=1,3,34,40$.

\subsection{The Five-Point Finite-Difference Scheme}

Here we illustrate the behavior of the five-point finite-difference scheme in(5.5). The numerical solution and instantaneous energy spectrum depicted in Figures 7.9 and 7.10, respectively, illustrate that the mechanism of energy transfer for the five-point scheme in (5.5) is qualitatively similar to the Kruskal-Zabusky finite-difference scheme discussed previously. The snapshots of the numerical solution computed with the five-point scheme shown in Figure 7.9 are similar to the snapshots of the solution in Figure 7.4. In particular, in contrast with the Fourier Galerkin truncation, when the energy is transfered to higher modes the numerical solution becomes more oscillatory near the shock. The energy transfer is a little bit slower for the five-point scheme compared with the Kruskal-Zabusky scheme. At time $t=1.6$ the numerical solution in Figure 7.4 (Kruskal-Zabusky scheme) is more oscillatory than the numerical solution computed with the five-point scheme presented in Figure 7.9. This is also reflected in the instantaneous energy spectrum, which is distinctly peaked at high wavenumbers for the Kruskal-Zabusky scheme, but is flatter at high wavenumbers for the fivepoint scheme. 

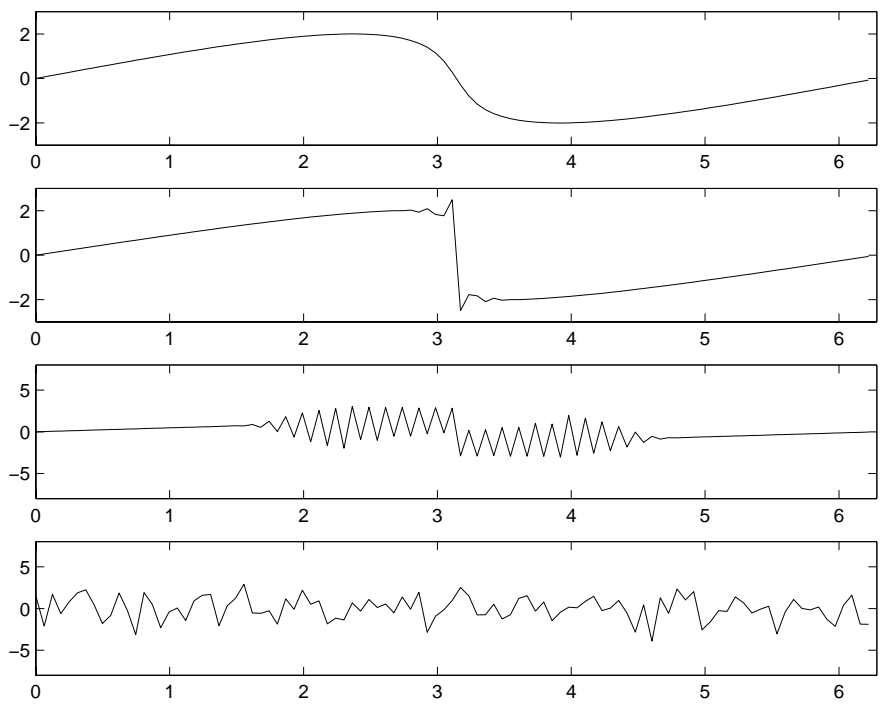

Figure 7.4: Kruskal-Zabusky Scheme with $N=50, \beta=50.5, u_{0}=2 \sin (x)$; Numerical Solution at time $t=0.4,0.6,1.6,20$.

The energy spectrum computed with the five-point finite-difference scheme is depicted in Figure 7.11. Similar to the simulations with the Kruskal-Zabusky scheme we obtain a reasonably good agreement with the prediction of equilibrium statistical mechanics. The largest errors are accumulated near the wavenumber $k^{r}=34 \approx(2 N+1) / 3$, but do not exceed $5.5 \%$. Correlation functions for wavenumbers $k=1,3,34,40$ and correlation times are presented in Figures 7.12 and 7.13, respectively. The wavenumber $k^{r}=34 \approx(2 N+1) / 3$ plays a special role for the five-point scheme and will be discussed later in this section. The rate of decay of the correlation function for Re $\hat{u}_{34}$ is much slower than for the Re $\hat{u}_{1}$, which is reflected in a sharp peak in the graph of correlation times in Figure 7.13 at $k=34$. This phenomena is probably caused by a resonance between the nonlinear interaction terms and aliasing terms in the equations in (6.26). We found that the resonant wavenumber $k^{r}$ scales with $N$ and the formula

$$
k^{r} \approx \frac{2 N+1}{3}
$$

holds for all the regimes we have tested. To demonstrate this, we present numerical results for the simulations with $N=300$. For $N=300$, the 

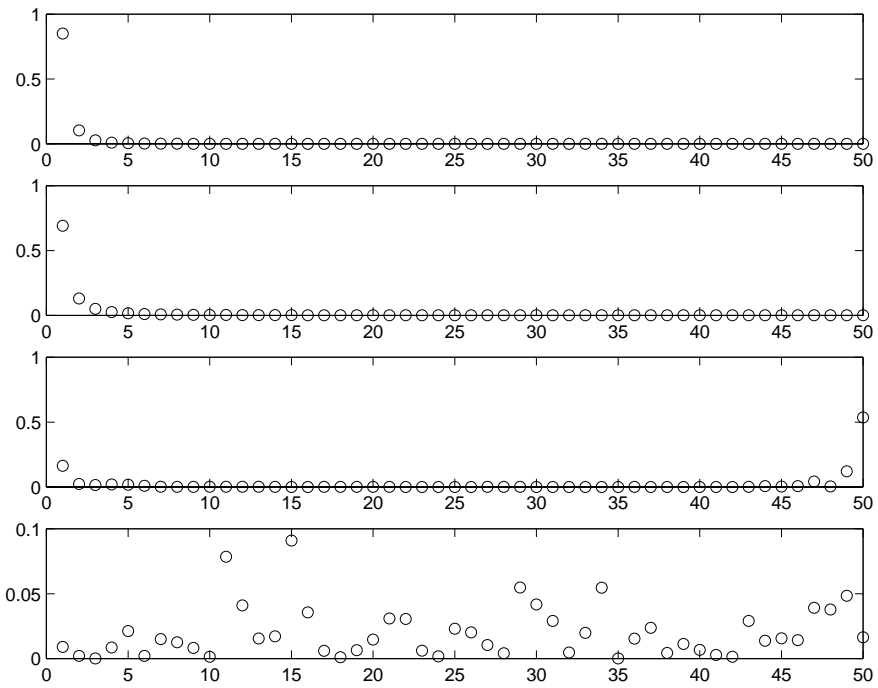

Figure 7.5: Kruskal-Zabusky Scheme with $N=50, \beta=50.5, u_{0}=2 \sin (x)$; Instantaneous Spectrum at time $t=0.4,0.6,1.6,20$.

resonant frequency in (7.2) is $k^{r}=200$. The dependence of correlation times on the wavenumbers for $N=300$ is depicted in Figure 7.14.

Similar to the simulation with $N=50$, Fourier modes with $k \approx k^{r}$ have the largest correlation times and the position of the peak in Figure 7.14 is shifted to $k^{r}=200$ according to (7.2).

We also found that in simulations with the five-point scheme correlation times for low wavenumbers decay faster than predicted by the scaling theory for correlations in (3.11). In particular, correlations times seem to obey a different power law

$$
T_{k} \sim k^{-3 / 2} .
$$

Log-log plot of correlation times for the 30 first low Fourier modes versus the wavenumbers for the simulations with $N=300$ is presented in Figure 7.15. A comparison with the same plots for the Fourier Galerkin truncation is also given on the same Figure. The solid line in Figure 7.15 corresponds to the power law $k^{-3 / 2}$ and the dashed line corresponds to the power law $k^{-1}$. Clearly, correlation times for low wavenumbers, $1 \leq k<10$, decay according to the power law $k^{-3 / 2}$ for the five-point scheme. For the Fourier Galerkin truncation (see Figure 7.15) correlations for the large scale 

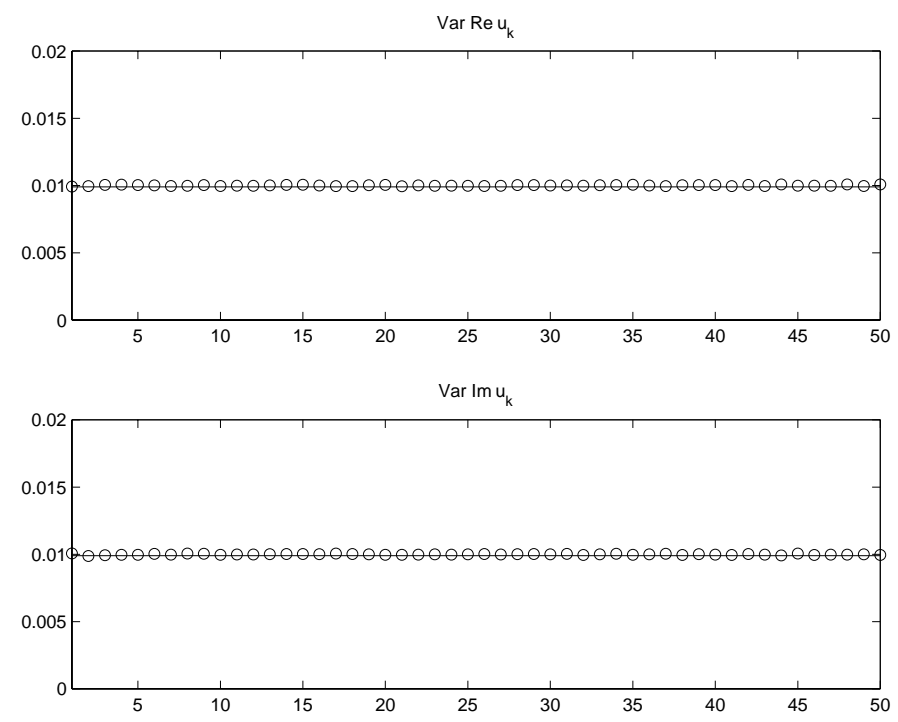

Figure 7.6: Kruskal-Zabusky Scheme with $N=50, \beta=50.5, u_{0}=2 \sin (x)$; Energy Spectrum; Circles - DNS, Solid Line - Canonical Predictions.

wavenumbers, $1 \leq k<30$, decay according to the $k^{-1}$ power law predicted by the scaling theory.

The discrepancy between the numerical and analytical estimates for the rate of decay of correlation functions for the five-point difference scheme might be explained by the failure of exact equipartition of energy for the modes around $k=k^{r}$. As we noted earlier, the largest discrepancies for the energy spectrum are concentrated at the wavenumber $k^{r}$.

To analyze the role of the wavenumber $k^{r}=(2 N+1) / 3$ we consider special steady state solutions of the five-point and the Kruskal-Zabusky schemes. It is easy to construct large families of steady state solutions of the five-point difference scheme in (5.5) following the discussion in Section 5.2 for the Goodman-Lax scheme. Define the grid values, $u_{j}^{0}$, to satisfy

$$
\begin{aligned}
& u_{3 k}^{0}=0 \\
& u_{3 k+1}^{0} u_{3 k+2}^{0}=Q_{0}
\end{aligned}
$$

with $Q_{0}$ a fixed constant. An explicit calculation establishes that these values define a steady state of the five-point difference scheme in (5.5). For the 

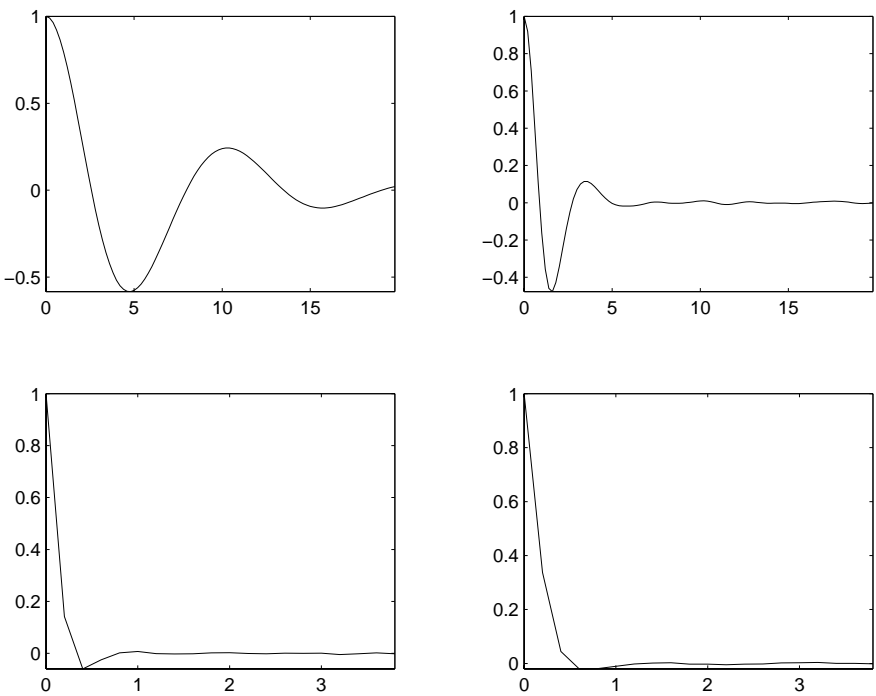

Figure 7.7: Kruskal-Zabusky Scheme with $N=50, \beta=50.5, u_{0}=2 \sin (x)$; Correlation Functions for Re $\hat{u}_{k}$ with $k=1,3,34,40$. Note that the correlation functions for Re $\hat{u}_{k}$ with $k=1,3$ oscillate through zero.

special case with $u_{3 k+1}^{0}=a_{1}, u_{3 k+2}^{0}=a_{2}$ independent of $k$ and $a_{1}, a_{2}$ arbitrary constants, such solutions are periodic with period $3 \Delta x$. Such steady states have a unique non-zero discrete Fourier coefficient $\hat{u}_{k^{r}}^{0}$ associated with the given mesh, $\Delta x$, if and only if for some integer $k^{r}$ with $\left|k^{r}\right| \leq N$, $k^{r}=(2 N+1) / 3$, i.e. $k^{r}$ is exactly the wavenumber associated with the resonant value observed in the simulations. The existence of these steady states provides a simple link between theory and simulations. However, for such steady states to provide an explanation of what is actually observed in the simulations, they need to be nonlinearly stable.

We comment here that the stability of such steady states for energy conserving difference schemes can be quite subtle. Recall from the beginning of Section 5.3, that we motivated the Kruskal-Zabusky difference scheme through the requirement that simple solutions exhibiting nonlinear instability are suppressed. That argument demonstrates that there are similar simple "resonant" steady state solutions of the Kruskal-Zabusky scheme 


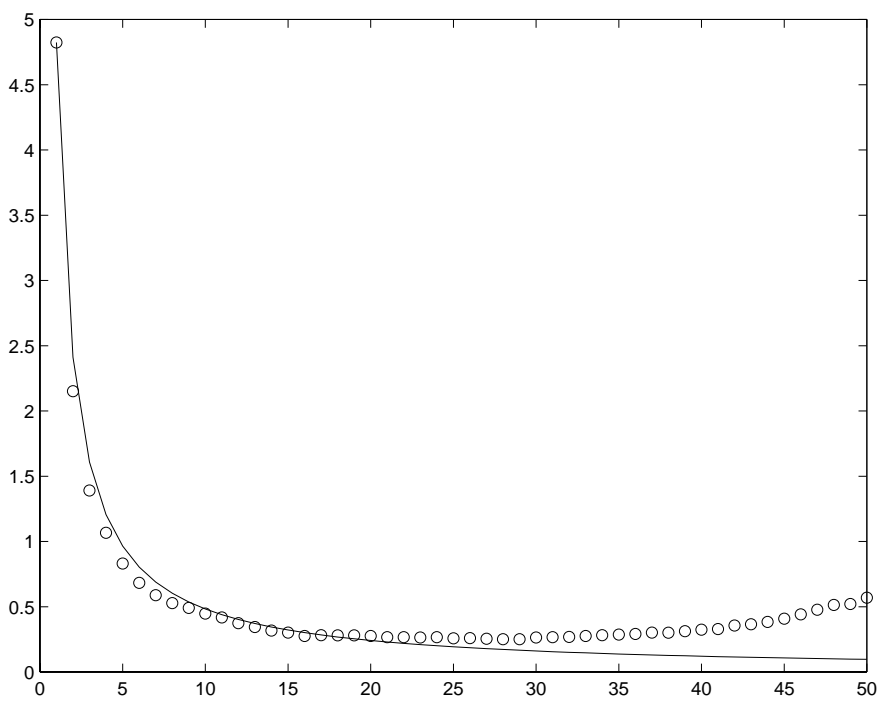

Figure 7.8: Kruskal-Zabusky Scheme with $N=50, \beta=50.5, u_{0}=2 \sin (x)$; Correlation Times; Circles - DNS, Solid Line - Predictions of the Scaling Theory.

with period $3 \Delta x$ of the form,

$$
\begin{aligned}
& u_{3 k}^{0}=0 \\
& u_{3 k+1}^{0}=a \\
& u_{3 k+2}^{0}=-a
\end{aligned}
$$

where $a$ is an arbitrary constant. The numerical evidence presented above suggests that these steady states play no role in the general dynamics of the Kruskal-Zabusky difference scheme and are nonlinearly unstable in contrast to the conjectured stability of those steady states for the five-point difference scheme. 

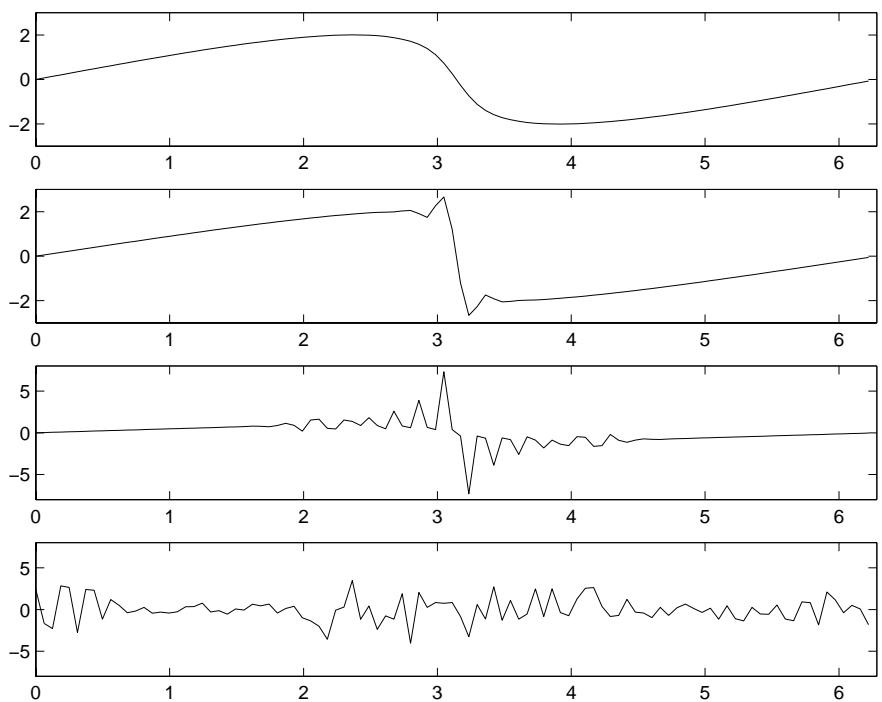

Figure 7.9: 5 -Point Scheme with $N=50, \beta=50.5, u_{0}=2 \sin (x)$; Numerical Solution at time $t=0.4,0.6,1.6,20$.

We address the issue about the stability of the steady states in (7.3) and (7.4) numerically and perform two numerical experiments for the fivepoint and the Kruskal-Zabusky schemes with $N=100$ and small perturbation of the initial data

$$
\begin{array}{r}
u_{3 k}^{0}=0 \\
u_{3 k+1}^{0}=2.42 \\
u_{3 k+2}^{0}=-2.42 .
\end{array}
$$

The energy of this steady state is $E=1.952$. We perturb the initial condition in (7.5) in Fourier space. The energy of perturbations is $1.9 \%$, so that the total energy in the simulations is $E_{t o t}=1.98$ and $\beta \approx 50.51$.

Time evolution of the Fourier coefficients with $k=1, k=66, k=k^{r}=$ 67 and $k=68$ for the five-point scheme and the Kruskal-Zabusky scheme are presented in Figures 7.16 and 7.17, respectively. Fourier coefficients with $k \neq k^{r}$ for the five-point scheme do not grow in time and are $O\left(10^{-4}\right)$ over the whole length of the numerical simulation and Fourier coefficient with $k=k^{r}$ settles to a steady state with a value close to the steady state in (7.5). 

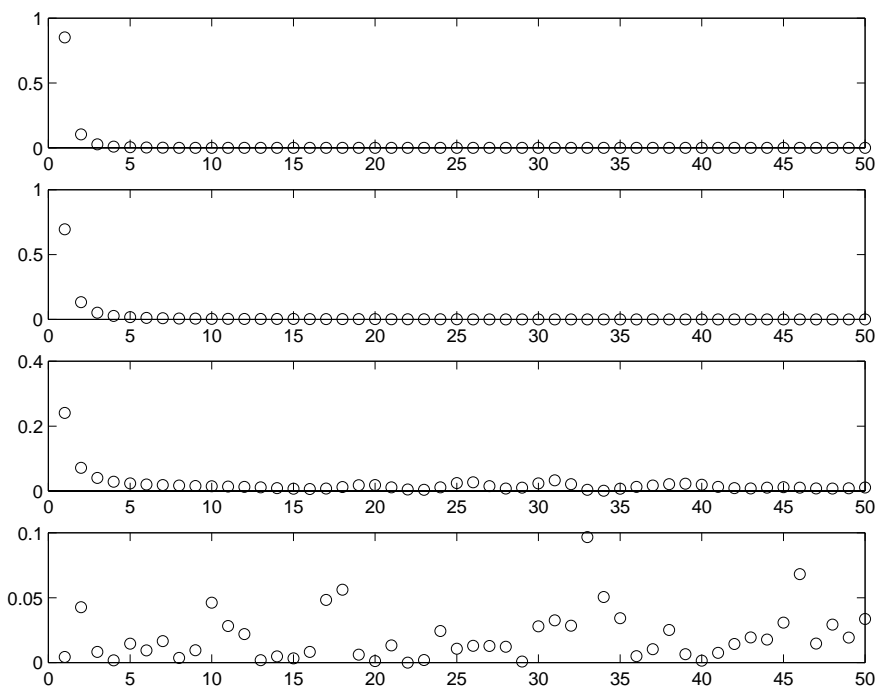

Figure 7.10: 5 -Point Scheme with $N=50, \beta=50.5, u_{0}=2 \sin (x)$; Instantaneous Spectrum at time $t=0.4,0.6,1.6,20$.

The instantaneous spectrum in this simulation always has $k^{r}$ as the dominant wavenumber. On the other hand, we observe very rapid energy transfer from $\hat{u}_{k^{r}}$ to other modes for the Kruskal-Zabusky scheme. The Fourier coefficient $\hat{u}_{k^{r}}$ decays fast for the simulation with the Kruskal-Zabusky scheme and by time $t=5$ the instantaneous spectrum looks completely random. Therefore, numerical evidence indicate that steady states in (7.5) are indeed nonlinearly stable for the five-point scheme and cause energy discrepancies for the mode with $k=k^{r}$ as well extremely long correlations for a few Fourier modes with $k \approx k^{r}$.

\section{Concluding Discussion and Future Directions}

In the previous sections, the authors have introduced simple models for onedimensional dynamics which are suitable approximations of the BurgersHopf equation in (1.1) involving either the Galerkin projection on Fourier modes or suitable finite-difference approximations which conserve both momentum and energy. Unlike the celebrated dissipative and dispersive approximations to (1.1) which exhibit exactly solvable and/or completely integrable behavior, the numerical evidence presented here indicates strong 

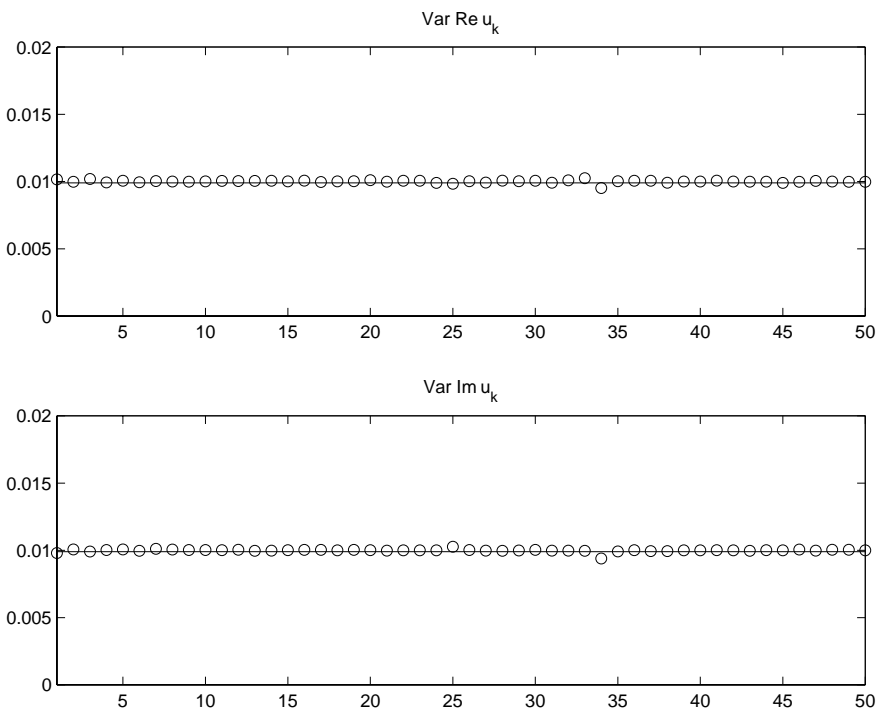

Figure 7.11: 5 -Point Scheme with $N=50, \beta=50.5, u_{0}=2 \sin (x)$; Energy Spectrum; Circles - DNS, Solid Line - Canonical Predictions.

intrinsic chaos with ergodic behavior in the models. Furthermore, the mathematical structure of the models including conservation of momentum, energy and the Liouville property, allows for simple statistical predictions for the models which are strongly confirmed by the numerical experiments. The models exhibit intrinsic slower decay of temporal correlations at larger scales and the increased predictability of the larger scale motions. This is one of the main achievements of the present paper. Furthermore, simple scaling theories for the behavior of the correlations have been developed and these predictions are also supported and confirmed by the numerical evidence reported here provided there are sufficiently many degrees of freedom. There are also some amusing discrepancies for the five-point difference scheme which are documented at the end of Section 7 .

Several intriguing issues regarding the models are worth pursuing in the future. The equation in (1.1) also conserves the higher invariants, $\int|u|^{p}$. Thus, are there suitable approximations to (1.1) which conserve momentum, energy and a discrete version of at least one of the higher invariants and also have the Liouville property with a well-defined statistical theory? 

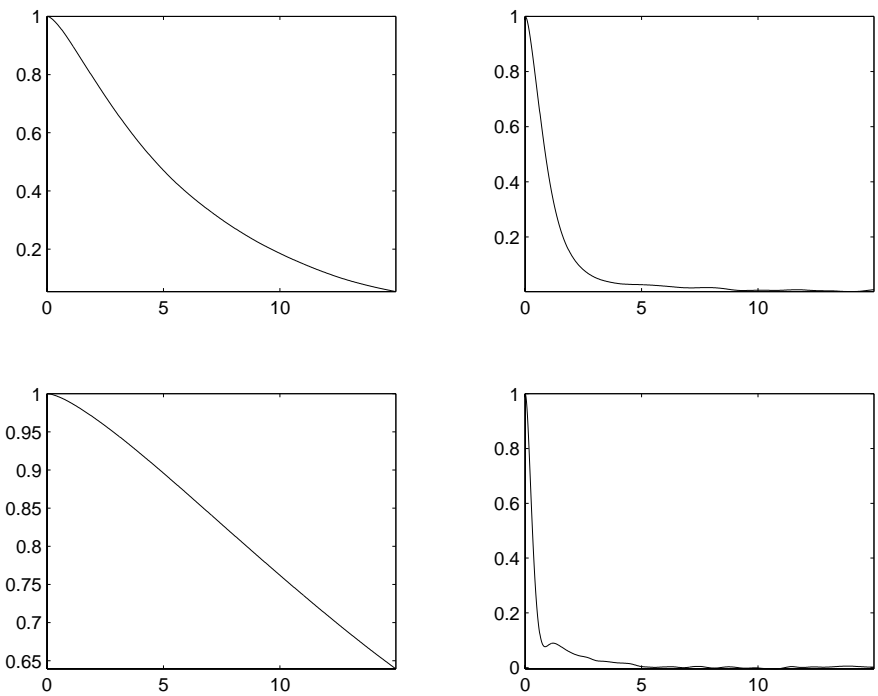

Figure 7.12: 5 -Point Scheme with $N=50, \beta=50.5, u_{0}=2 \sin (x)$; Correlation Functions for Re $\hat{u}_{k}$ with $k=1,3,34,40$. Note the extremely slow decay of correlations at $k=34$.

The original motivation of the "toy" models developed here is to utilize these models to check reduced stochastic modeling procedures for more complex physical or biological models with a range of correlations on a simple unambiguous one-dimensional model system that exhibits such behavior. Cai, Vanden Eijnden, and one of the authors have done this for the model and report on this work elsewhere [15].

Spectrally truncated approximation for idealized geophysical flows that conserve both energy and enstrophy play an important role in building idealized climate models [16], [17] where various facets of observational as well as computational [18] and stochastic [6], [7] modeling phenomena can be checked in a relatively unambiguous context. The even simpler onedimensional models proposed here have the potential to provide more mathematical insight on a variety of the issues encountered in these problems. In that context, as discussed by Leith [16], an important issue is the validity of the fluctuation-dissipation theorem. It is very interesting to test both the fluctuation-dissipation theorem and various practical predictability strategies for the models in this paper with a wide range of scaling behavior for 


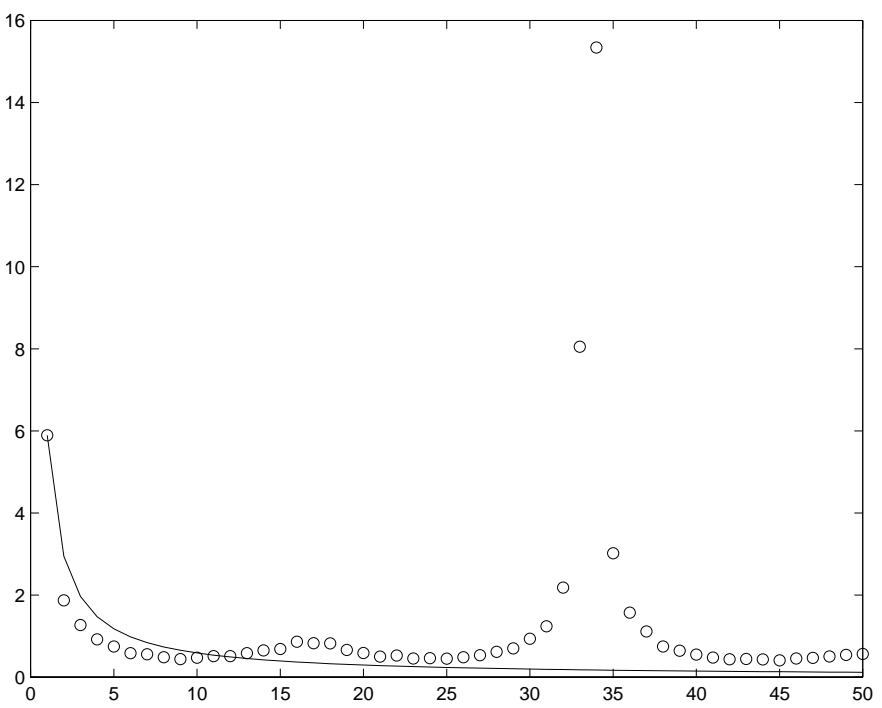

Figure 7.13: 5 -Point Scheme with $N=50, \beta=50.5, u_{0}=2 \sin (x)$; Correlation Times; Circles - DNS, Solid Line - Predictions of the Scaling Theory.

correlations. The authors plan to address these questions for the models in this paper with other collaborators in the near future.

There is another discrete model system of ODE's, the OrszagMcLaughlin model [24] given by the finite-difference scheme

$$
\frac{d u_{j}}{d t}=u_{j+1} u_{j+2}+u_{j-1} u_{j-2}-2 u_{j+1} u_{j-1}
$$

which conserves energy, but not momentum. Clearly this model is not consistent with the Burgers-Hopf equation in (1.1) or any other differential equation in any sense. Nevertheless, this model has chaotic dynamics and Carnevale, et al [25] have utilized the model to provide an interesting test of the fluctuation-dissipation theorem. It is unclear whether these models exhibit a wide range of scaling behavior for correlations. Thus, it is also interesting to compare the statistical behavior in these models with that in the new models presented in this paper. The authors plan to do this in the near future.

ACKnowledgment. The research of Andrew Majda is partially supported by NSF Grant DMS-9972865, ONR Grant N00014-96-1-0043, and ARO 


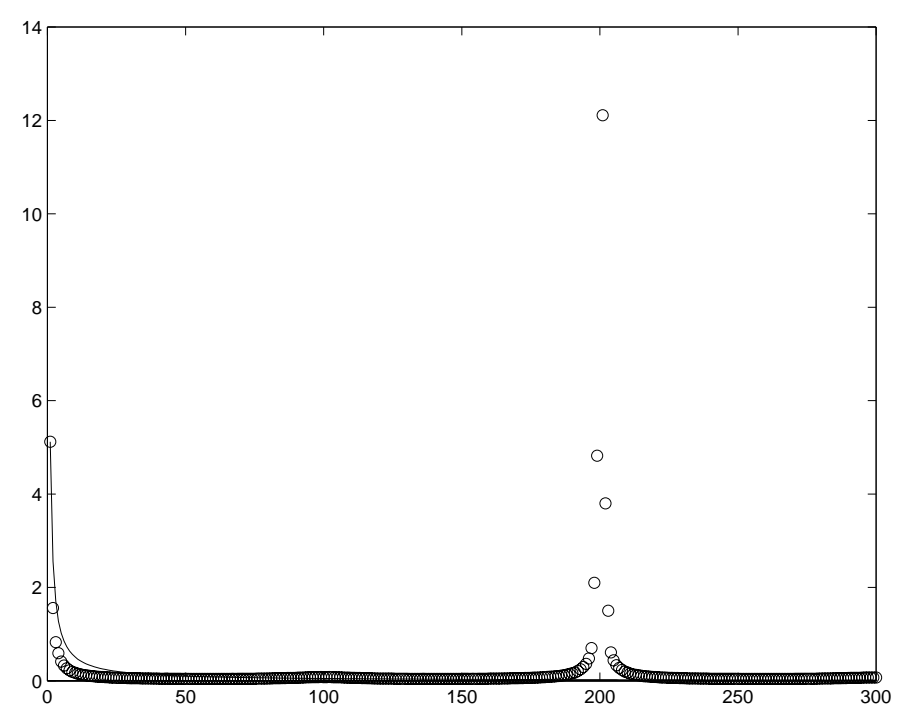

Figure 7.14: 5 -Point Scheme with $N=300, \beta=50.51$, Random IC; Correlation Times; Circles - DNS, Solid Line - Predictions of the Scaling Theory.

Grant DAAG55-98-1-0129. Ilya Timofeyev is supported as a postdoctoral fellow by NSF Grant DMS-9972865 and ARO Grant DAAG55-98-1-0129.

\section{References}

[1] R. Kleeman and A. Moore, J. Atmos. Sci. 54 (1997), 753-767.

[2] R. Saravanan and J. McWilliams, J. Climate 10 (1997), 1114-1127.

[3] G. Branstator, J. Atmos. Sci. 52 (1995), 207-226.

[4] Ch. Schutte, A. Fisher, W. Huisinga and P. Deuflhard, J. Comp. Phys. 151 (1999), 146-168.

[5] H. Gordon and R. Somoraj, Proteins Struct. Funct. Genesis 14 (1992), 249-262.

[6] A. Majda, I. Timofeyev and E. Vanden Eijnden, Proc. Nat. Acad. Sci. 96 (1999), 14687-14691.

[7] A. Majda, I. Timofeyev and E. Vanden Eijnden, A mathematical framework for Stochastic Climate Models, Comm. Pure Appl. Math. 54 (2001), 892 974. 


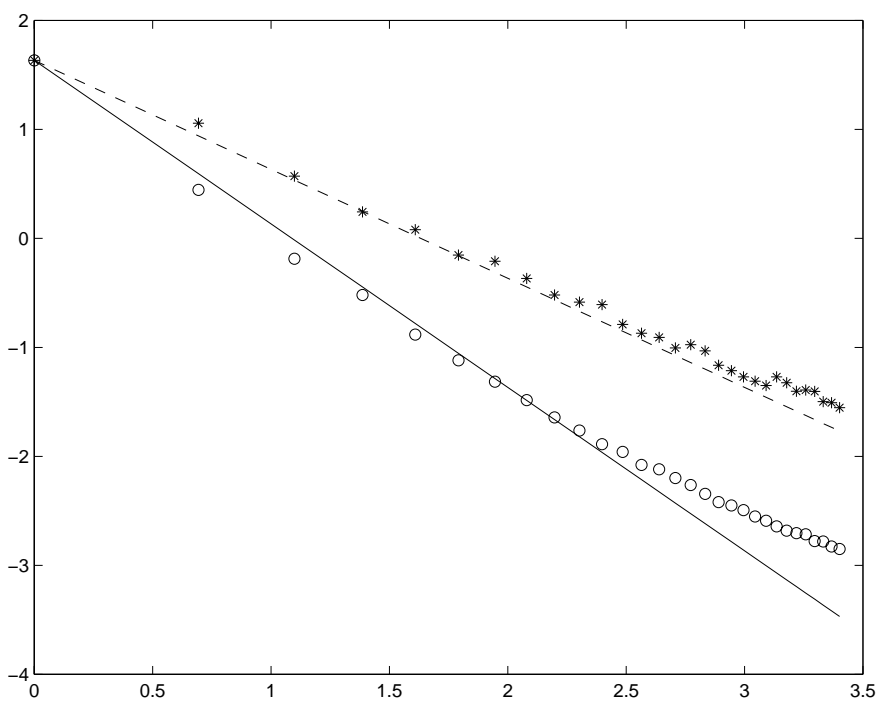

Figure 7.15: Log-Log Plot of Correlation Times for 30 first Fourier modes. Circles - Numerics for the 5-Point Scheme with $N=300, \beta=50.51$; Stars Numerics for the Galerkin truncation with $\Lambda=50, \beta=50$; Solid Line - Power Law $k^{-3 / 2}$; Dashed Line - Power Law $k^{-1}$.

[8] G.B. Whitham, Linear and Nonlinear Waves, Academic Press, New York, 1974.

[9] J.M. Burgers, Adv. Appl. Mech. 1 (1948), 171-199.

[10] E. Hopf, Comm. Pure Appl. Math. 3 (1950), 201-230.

[11] C.S. Gardner, J.M. Greene, M.D. Kruskal and R.M. Miura, Phys. Rev. Lett. 19 (1967), 1095-1097.

[12] J. Goodman and P.D. Lax, Comm. Pure Appl. Math. 41 (1988), 591-613.

[13] J. Pedlosky, Geophysical Fluid Dynamics, Springer-Verlag, New York, 1982, Sec. 7.16, 532-535.

[14] N. Zabusky and M.D. Kruskal, Phys. Rev. Lett. 15 (1965), 240-243.

[15] D. Cai, A. Majda And E. Vanden Eijnden, Stochastic modeling of nonlinear dynamics with intrinsic stochasticity and scaling behavior, preprint, $1 / 10 / 2000$.

[16] C. E. Leith, J. Atmos. Sci. 32 (1975), 2022-2025.

[17] G. Carnevale and J. Frederiksen, J. Fluid Mech. 175 (1987), 157-181.

[18] J. Frederiksen, M. Dix And S. Kepert, J. Atmos. Sci. 53 (1996), 8871004. 


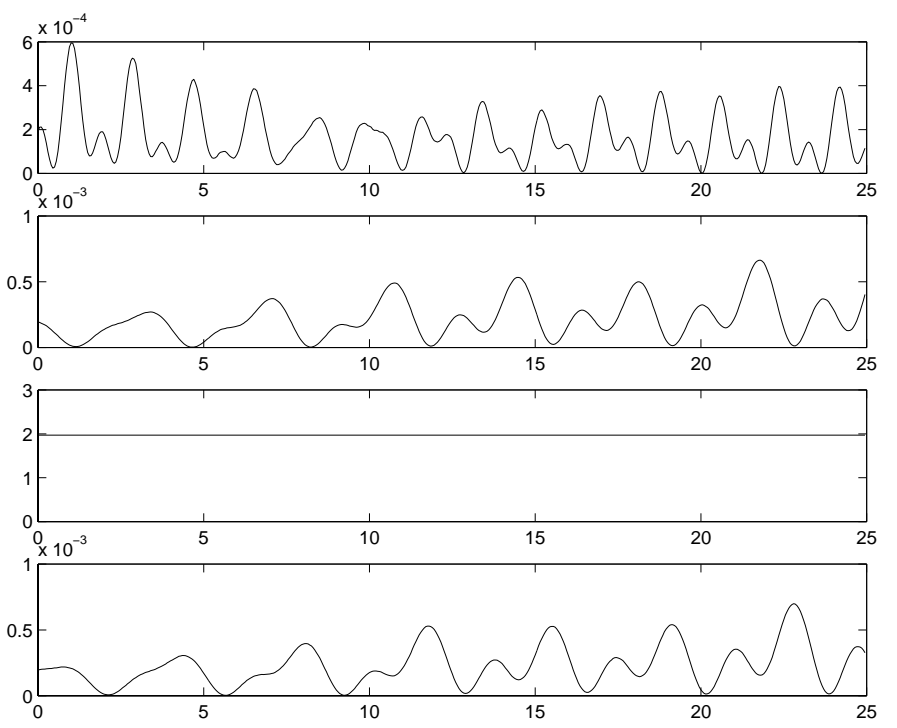

Figure 7.16: 5-Point Scheme; Simulations with $N=100, \beta=50.51$; Perturbation of the Stationary Solution in (7.5). Time evolution of $\left|\hat{u}_{k}\right|^{2}$ with $k=1$, 66, 67 and 68. Note that the perturbation amplitudes for $k=1,66$ and 68 are $O\left(10^{-4}\right)$ and evolve slowly in time while the mode with $k=67$ is timeindependent.

[19] A. Majda And I. Timofeyev, Remarkable Statistical Behavior for Truncated Burgers-Hopf Dynamics, Proc. Nat. Acad. Sci. 97 (23) (2000), 12413-12417.

[20] D. Levermore and J.G. Liu, Physica D 99 (1996), 191-216.

[21] A. Harten, Recent developments in shock-capturing schemes in Proceedings of the International Congress of Mathematics, Kyoto, 1990, 1549-1560.

[22] A. MAJDA, Real world turbulence and modern applied mathematics, in Mathematics: Frontiers and Perspectives 200099 (2000), 137-151.

[23] A. Lasota And M. Mackey, Chaos, Fractals, and Noise, Applied Math. Sciences 97, Springer-Verlag, New York, 1994, Chapter 9.

[24] S. Orszag and J. McLaughlin, Physica D 68 (1980).

[25] G. Carnevale, M. Falcioni, S. Isola, R. Purini and A. Vulpiani, Phys. Fluids A 3 (1991), 2247-2254. 

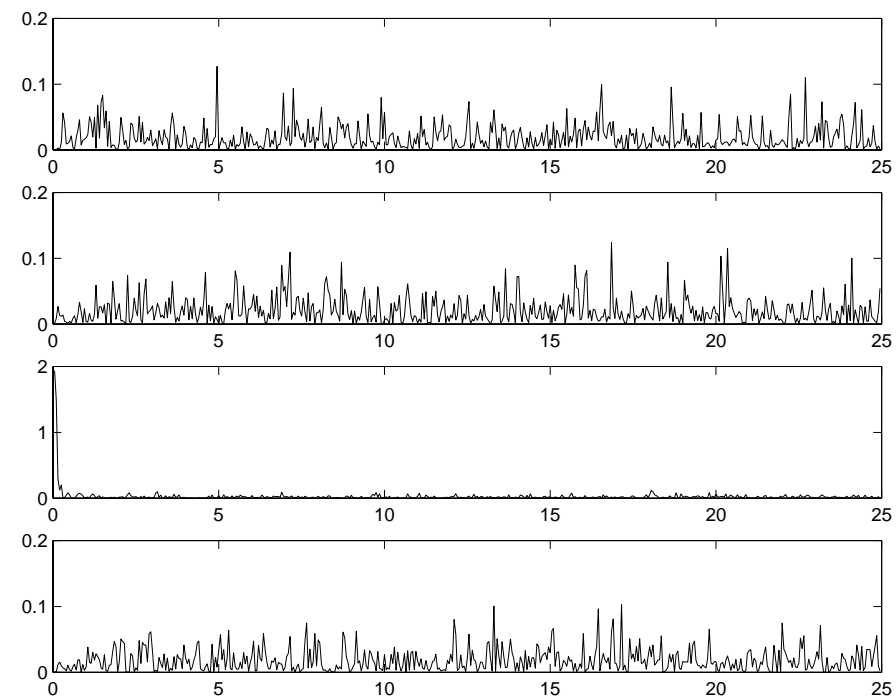

Figure 7.17: Kruskal-Zabusky Scheme; Simulations with $N=100, \beta=50.51$; Perturbation of the Stationary Solution in (7.5). Time evolution of $\left|\hat{u}_{k}\right|^{2}$ with $k=1,66,67$ and 68 . Note that the mode with $k=67$ has lost all its energy while the other three modes have amplitudes $O\left(10^{-1}\right)$.

A. Majda and I. Timofeyev

Courant Institute of Mathematical Sciences

New York University

251 Mercer Street

New York, NY 10012, USA

To access this journal online:

(40) http://www.birkhauser.ch 